\title{
Low-Complexity Scheduling Policies for Achieving Throughput and Asymptotic Delay Optimality in Multi-Channel Wireless Networks
}

\author{
Bo Ji, Gagan R. Gupta, Xiaojun Lin, and Ness B. Shroff
}

\begin{abstract}
In this paper, we study the scheduling problem for downlink transmission in a multi-channel (e.g., OFDM-based) wireless network. We focus on a single cell, with the aim of developing a unifying framework for designing low-complexity scheduling policies that can provide optimal performance in terms of both throughput and delay. We develop new easy-to-verify sufficient conditions for rate-function delay optimality (in the many-channel many-user asymptotic regime) and throughput optimality (in general non-asymptotic setting), respectively. The sufficient conditions allow us to prove rate-function delay optimality for a class of Oldest Packets First (OPF) policies and throughput optimality for a large class of Maximum Weight in the Fluid limit (MWF) policies, respectively. By exploiting the special features of our carefully chosen sufficient conditions and intelligently combining policies from the classes of OPF and MWF policies, we design hybrid policies that are both rate-function delay-optimal and throughput-optimal with a complexity of $O\left(n^{2.5} \log n\right)$, where $n$ is the number of channels or users. Our sufficient condition is also used to show that a previously proposed policy called Delay Weighted Matching (DWM) is rate-function delay-optimal. However, DWM incurs a high complexity of $O\left(n^{5}\right)$. Thus, our approach yields significantly lower complexity than the only previously designed delay and throughput optimal scheduling policy. We also conduct numerical experiments to validate our theoretical results.
\end{abstract}

\section{INTRODUCTION}

Designing high-performance scheduling algorithms has been a vital and challenging problem in wireless networks. Among the many dimensions of network performance, the most critical ones are perhaps throughput, delay, and complexity. However, it is in general extremely difficult, if not impossible, to

B. Ji is with AT\&T Labs. X. Lin is with School of ECE at Purdue University. N. B. Shroff is with Departments of ECE and CSE at the Ohio State University. Emails: ji.33@osu.edu, gagan.gupta@iitdalumni.com, linx@ecn.purdue.edu, shroff.11@osu.edu. 
develop scheduling policies that attain the optimal performance in terms of both throughput and delay, without the cost of high complexity [1].

In this paper, we focus on the setting of a single-hop multi-user multi-channel system. A practically important example of such a multi-channel system is the downlink of a single cell in 4G OFDM-based celluar networks (e.g., LTE and WiMax). Such a system typically has a large bandwidth that can be divided into multiple orthogonal sub-bands (or channels), which need to be allocated to a large number of users by a scheduling algorithm. The main question that we will attempt to answer in this paper is the following: How do we design efficient scheduling algorithms that simultaneously provide high throughput, small delay, and low complexity?

We consider a multi-channel system that has $n$ channels and a proportionally large number of users. This setting is referred to as the many-channel many-user asymptotic regime when $n$ goes infinity. The connectivity between each user and each channel is assumed to be time-varying, due to channel fading. We assume that the base station (BS) maintains separate First-in First-out (FIFO) queues that buffer the packets destined to each user. The delay metric that we will focus on in this paper is the asymptotic decay-rate (also called the rate-function in the large-deviations theory) of the probability that the largest packet waiting time in the system exceeds a fixed threshold, as both the number of channels and the number of users go to infinity. (Refer to Eq. (2) for the precise definition.)

Next, we overview some key related works. In [2], the authors considered a single-server model with time-varying channels, and showed that the longest-connected-queue (LCQ) algorithm minimizes the average delay for the special case of symmetric (i.i.d. Bernoulli) arrival and channel. Later, the results were generalized for a multi-server model in [3]. The authors of [4] further generalized the multi-server model by considering more general permutation-invariant arrivals (that are not restricted to Bernoulli only) and multi-rate channel model. Hence, the problem of minimizing a general cost function of queue-lengths (includes minimizing the expected delay) studied in [4] becomes harder. There, for special cases of ONOFF channel model with two users or allowing for fractional server allocation, an optimal scheduling algorithm was derived. Using the insights obtained from the analytical results in [4] for ON-OFF channel model, in [5] the same authors developed heuristic policies and showed through simulations that their proposed heuristic policies perform well under a general channel model. Note that in contrast to this paper, the above studies directly minimize queue-length or delay in a non-asymptotic regime, which is an extremely difficult problem in general.

As we do in this paper, another body of related works [6]-[9] focus on the many-channel many-user asymptotic regime, where the analysis may become more tractable. Even though the analysis for an 
asymptotic setting is very different from the non-asymptotic analysis in [4], it is remarkable that some of the insights are consistent. For example, from a delay optimality perspective, the above two bodies of studies both point to the tradeoff between maximizing instantaneous throughput and balancing the queues. Thus, we believe that, collectively, these studies under different settings provide useful insights for designing efficient scheduling solutions in practice.

In [6]-[9], a number of queue-length-based scheduling policies for achieving optimal or positive queuelength-based rate-unction 1 were developed. In particular, an optimal scheduling policy that maximizes the queue-length-based rate-function has been derived with complexity $O\left(n^{3}\right)$ [9]. However, these works have two key limitations. First, the schedulers' performance are proven under the assumption that the arrival process is i.i.d. not only across users, but also in time, which does not model the temporal correlation present in most real network traffic. More importantly, it is well known that good queue-length performance does not necessarily translate to good delay performance [10]-[12]. A recently developed scheduling policy called Delay Weighted Matching (DWM) [10], [11], which makes scheduling decisions by maximizing the sum of the delays of the scheduled packets in each time-slot, focuses directly on the delay performance as we do in this paper. It has been shown that the DWM policy is rate-function delayoptimal in some cases. However, DWM has the following two key drawbacks: 1) it is unclear whether DWM is rate-function delay-optimal in general; and 2) DWM yields a very high complexity of $O\left(n^{5}\right)$ and is thus not amenable for practical implementations.

Hence, the state-of-the-art does not satisfactorily answer our main question of how to design scheduling policies with a low complexity, while guaranteeing provable optimality for both throughput and delay. In this paper, we address this challenge, and provide the following key intellectual contributions.

First, we characterize easy-to-verify sufficient conditions for rate-function delay optimality in the manychannel many-user asymptotic regime and for throughput optimality in general non-asymptotic settings. The sufficient conditions allow us to prove rate-function delay optimality for a class of Oldest Packets First $(O P F)$ policies and throughput optimality for a large class of Maximum Weight in the Fluid limit $(M W F)$ policies. Moreover, the sufficient conditions can be used to show that a slightly modified version of the DWM policy is both rate-function delay-optimal and throughput-optimal.

Second, we develop an $O\left(n^{2.5} \log n\right)$-complexity scheduling policy called DWM- $n$. The DWM- $n$ policy shares the high-level similarity with the DWM policy [10], [11], but makes scheduling decisions in each

\footnotetext{
${ }^{1}$ The queue-length-based rate-function is defined as the asymptotic decay-rate of the probability that the largest queue length in the system exceeds a fixed threshold.
} 
time-slot by maximizing the sum of the delays of the scheduled packets over only the $n$ oldest packets in the system, rather than over all the packets as in the DWM policy. We show that DWM- $n$ is an OPF policy and is thus rate-function delay-optimal. However, DWM- $n$ is not throughput-optimal in general, and may perform poorly when $n$ is not large.

Third, by exploiting the special features of our carefully-chosen sufficient conditions and intelligently combining policies from the classes of OPF and MWF policies, we develop a class of two-stage hybrid policies that are both throughput-optimal and rate-function delay-optimal. In particular, we can adopt the DWM- $n$ policy in stage 1 and the Delay-based MaxWeight Scheduling (D-MWS) policy in stage 2, respectively, so as to design an optimal hybrid policy with a low complexity of $O\left(n^{2.5} \log n\right)$.

Finally, we conduct numerical experiments to validate our theoretical results in different scenarios.

\section{SySTEM MODEL}

We consider a multi-channel system with $n$ orthogonal channels and $n$ users, which can be modeled as a multi-queue multi-server system with stochastic connectivity, as shown in Fig. 11 For ease of presentation, the number of users is assumed to be equal to the number of channels. Our analysis for rate-function delay optimality follows similarly if the number of users scales linearly with the number of channels. Throughout the rest of the paper, we will use the terms "user" and "queue" interchangeably, and use the terms "channel" and "server" interchangeably. We assume that time is slotted. In a time-slot, a server can be allocated to only one queue, but a queue can get service from multiple servers. The connectivity between queues and servers is time-varying, i.e., it can change between "ON" and "OFF" from time to time. We assume that perfect channel state information (i.e., whether each channel is ON or OFF for each user in each time-slot) is known at the BS. This is a reasonable assumption in the downlink scenario of a single cell in a multi-channel cellular system with dedicated feedback channels.

The notations used in this paper are as follows. We let $Q_{i}$ denote the FIFO queue (at the BS) associated with the $i$-th user, and let $S_{j}$ denote the $j$-th server. We assume infinite buffer for all the queues. Let $A_{i}(t)$ denote the number of packet arrivals to queue $Q_{i}$ in time-slot $t$, let $A(t)=\sum_{i=1}^{n} A_{i}(t)$ denote the cumulative arrivals to the entire system in time-slot $t$, and let $A\left(t_{1}, t_{2}\right)=\sum_{\tau=t_{1}}^{t_{2}} A(\tau)$ denote the cumulative arrivals to the system from time $t_{1}$ to $t_{2}$. We let $\lambda_{i}$ be the mean arrival rate of queue $Q_{i}$, and let $\lambda \triangleq\left[\lambda_{1}, \lambda_{2}, \ldots, \lambda_{n}\right]$ denote the arrival rate vector. We assume that packet arrivals occur at the beginning of each time-slot, and packet departures occur at the end of each time-slot. We let $Q_{i}(t)$ denote the length of queue $Q_{i}$ at the beginning of time-slot $t$ immediately after packet arrivals. Also, let $Z_{i, l}(t)$ denote the delay (i.e., waiting time) of the $l$-th packet of queue $Q_{i}$ at the beginning of time-slot $t$, which 


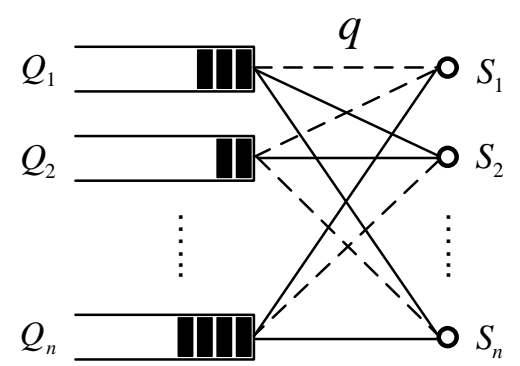

Fig. 1. System model. The connectivity between each pair of queue $Q_{i}$ and server $S_{j}$ is "ON" (denoted by a solid line) with probability $q$, and "OFF" (denoted by a dashed line) otherwise.

is measured since the time when the packet arrived to queue $Q_{i}$ until the beginning of time-slot $t$. Note that at the end of each time-slot, the packets still present in the system will have their delays increased by one due to the elapsed time. We then let $W_{i}(t)=Z_{i, 1}(t)$ denote the head-of-line (HOL) packet delay of queue $Q_{i}$ at the beginning of time-slot $t$. Further, we use $C_{i, j}(t)$ to denote the capacity of the link between queue $Q_{i}$ and server $S_{j}$ in time-slot $t$, i.e., the maximum number of packets that can be served by server $S_{j}$ from queue $Q_{i}$ in time-slot $t$. Finally, we let $\mathbb{1}_{\{\cdot\}}$ denote the indicator function, and let $\mathbb{Z}^{+}$ denote the set of positive integers.

We now state the assumptions on the arrival processes. The throughput analysis is carried out under very general conditions (Assumption 1] similar to that of [13].

Assumption 1: For each user $i \in\{1,2, \ldots, n\}$, the arrival process $A_{i}(t)$ is an irreducible and positive recurrent Markov chain with countable state space, and satisfies the Strong Law of Large Numbers: That is, with probability one,

$$
\lim _{t \rightarrow \infty} \frac{\sum_{\tau=0}^{t-1} A_{i}(\tau)}{t}=\lambda_{i}
$$

We also assume that the arrival processes are mutually independent across users (which can be relaxed for showing throughput optimality, as discussed in [13].)

Assumptions 2 and 3 (stated below) will be used for rate-function delay analysis.

Assumption 2: There exists a finite $L$ such that $A_{i}(t) \leq L$ for any $i$ and $t$, i.e., arrivals are bounded. Further, we assume $\mathbb{P}(A(s, s+t-1)=L n t)>0$ for any $s, t$ and $n$.

Assumption 3: The arrival processes are i.i.d. across users, and $\lambda_{i}=p$ for any user $i$. Given any $\epsilon>0$ and $\delta>0$, there exists $T_{B}(\epsilon, \delta)>0, N_{B}(\epsilon, \delta)>0$, and a positive function $I_{B}(\epsilon, \delta)$ independent of $n$ and $t$ such that

$$
\mathbb{P}\left(\frac{\sum_{\tau=1}^{t} \mathbb{1}_{\{|A(\tau)-p n|>\epsilon n\}}}{t}>\delta\right)<\exp \left(-n t I_{B}(\epsilon, \delta)\right)
$$


for all $t \geq T_{B}(\epsilon, \delta)$ and $n \geq N_{B}(\epsilon, \delta)$.

Assumptions 2 and 3 are relatively mild. The first part of Assumption 2 and Assumption 3 have also been used in the previous work [10], [11] for rate-function delay analysis. In Assumption 2] the first part requires that the arrivals in each time-slot have bounded support; and the second part guarantees that there is a positive probability that all users have the maximum number of arrivals in any time-interval with any length. Assumption 3 allows the arrivals for each user to be correlated over time (e.g., arrivals driven by a two-state Markov chain), which is more general than the arrival processes (i.i.d. in time) considered in [6]-[9].

We then describe our channel model as follows.

Assumption 4: In any time-slot $t, C_{i, j}(t)$ is modeled as a Bernoulli random variable with a parameter $q \in(0,1)$, i.e.,

$$
C_{i, j}(t)= \begin{cases}1, & \text { with probability } q, \\ 0, & \text { with probability } 1-q .\end{cases}
$$

All the random variables $C_{i, j}(t)$ are assumed to be mutually independent across all the variables $i, j$ and $t$.

We assume unit channel capacity as above. Under this assumption, we will also let $C_{i, j}(t)$ denote the connectivity between queue $Q_{i}$ and server $S_{j}$ in time-slot $t$, without causing confusions. As in the previous works [6]-[11], in this paper we assume i.i.d. channels for the analytical results only. Moreover, we will show through simulations that our proposed low-complexity solution also performs well in more general scenarios, e.g., when the channel condition follows a two-state Markov chain that allows correlation over time. Further, we will briefly discuss how to generalize our solution to more general scenarios towards the end of this paper.

Next, we define the optimal throughput region (or stability region) of the system for any fixed integer $n>0$. As in [13], a stochastic queueing network is said to be stable if it can be described as a discretetime countable Markov chain and the Markov chain is stable in the following sense: The set of positive recurrent states is nonempty, and it contains a finite subset such that with probability one, this subset is reached within finite time from any initial state. When all the states communicate, stability is equivalent to the Markov chain being positive recurrent. The throughput region of a scheduling policy is defined as the set of arrival rate vectors for which the network remains stable under this policy. Further, the optimal throughput region is defined as the union of the throughput regions of all possible scheduling policies. We let $\Lambda^{*}$ denote the optimal throughput region. A scheduling policy is throughput-optimal, if it can stabilize any arrival rate vector $\lambda$ strictly inside $\Lambda^{*}$. For more discussions on the characterization of $\Lambda^{*}$ 
please refer to Appendix A

For delay analysis, we consider the many-channel many-user asymptotic regime. Let $W(t)$ denote the largest HOL delay over all the queues (i.e., the largest or worst packet waiting time in the system) at the beginning of time-slot $t$, i.e., $W(t) \triangleq \max _{1 \leq i \leq n} W_{i}(t)$. Assuming that the system is stationary and ergodic, we define the rate-function for integer threshold $b \geq 0$ as

$$
I(b) \triangleq \lim _{n \rightarrow \infty} \frac{-1}{n} \log \mathbb{P}(W(0)>b) .
$$

We can then estimate $\mathbb{P}(W(0)>b) \approx \exp (-n I(b))$ when $n$ is large, and the estimation accuracy tends to be higher as $n$ increases. Clearly, for large $n$ a larger value of the rate-function leads to better delay performance, i.e., a smaller probability that the largest HOL delay exceeds a certain threshold. A scheduling policy is rate-function delay-optimal if for any fixed integer threshold $b \geq 0$, it achieves the maximum rate-function over all possible scheduling policies.

Note that the rate-function optimality is studied in the asymptotic regime, i.e., when $n$ goes to infinity. Although the convergence of the rate-function is typically fast, the throughput performance may be poor for small to moderate values of $n$. As a matter of fact, a rate-function delay-optimal policy may not even be throughput-optimal for a fixed $n$ (e.g., the DWM- $n$ policy that we will propose in Section IV]. To that end, we are interested in designing scheduling policies that maximize both the throughput (for any fixed $n$ ) and the rate-function (in the many-channel many-user asymptotic regime).

\section{AN UPPER BOUND ON THE RATE-FunCtion}

In this section, we derive an upper bound on the rate-function that can be achieved by any scheduling algorithm. Then, later in Section IV we will provide a sufficient condition for achieving this upper bound and thus achieving the optimal rate-function.

As in [10], [11], for any integer $t>0$ and any real number $x \geq 0$, we define the quantity

$$
I_{A}(t, x) \triangleq \sup _{\theta>0}\left[\theta(t+x)-\lambda_{A_{i}(-t+1,0)}(\theta)\right]
$$

where $\lambda_{A_{i}(-t+1,0)}(\theta)=\log \mathbf{E}\left[e^{\theta A_{i}(-t+1,0)}\right]$ is the cumulant-generating function of $A_{i}(-t+1,0)$ and $A_{i}(-t+1,0)=\sum_{\tau=-t+1}^{0} A_{i}(\tau)$. From Cramer's Theorem, this quantity, $I_{A}(t, x)$, is equal to the asymptotic decay-rate of the probability that in any interval of $t$ time-slots, the total number of packet arrivals to the system is no smaller than $n(t+x)$, as $n$ tends to infinity, i.e.,

$$
\lim _{n \rightarrow \infty} \frac{-1}{n} \log \mathbb{P}(A(-t+1,0) \geq n(t+x))=I_{A}(t, x) .
$$


Define the following for the case of $L>1$. For any integer $x \geq 0$, we define $t_{x}$ as

$$
t_{x} \triangleq \frac{x}{L-1}
$$

Then, we define $\Psi_{b} \triangleq\left\{c \in\{0,1, \ldots, b\} \mid t_{b-c} \in \mathbb{Z}^{+}\right\}$. It will later become clear why the values of $c$ in the set $\Psi_{b}$ are important and need to be considered separately. Let $I_{X} \triangleq \log \frac{1}{1-q}$. Then, for any integer $b \geq 0$, we define the quantity

$$
\begin{aligned}
& I_{0}(b) \triangleq \min \left\{(b+1) I_{X},\right. \\
& \min _{0 \leq c \leq b}\left\{\inf _{t>t_{b-c}} I_{A}(t, b-c)+c I_{X}\right\}, \\
&\left.\min _{c \in \Psi_{b}}\left\{I_{A}\left(t_{b-c}, b-c\right)+(c+1) I_{X}\right\}\right\} .
\end{aligned}
$$

Further, for any given integer $L \geq 1$, we define

$$
I_{0}^{*}(b) \triangleq \begin{cases}(b+1) I_{X}, & \text { if } L=1 \\ I_{0}(b), & \text { if } L>1\end{cases}
$$

In the following theorem, we show that for any given integer threshold $b \geq 0, I_{0}^{*}(b)$ is an upper bound of the rate-function that can be achieved by any scheduling policy.

Theorem 1: Given the system model described in Section \. for any scheduling algorithm, we have

$$
\limsup _{n \rightarrow \infty} \frac{-1}{n} \log \mathbb{P}(W(0)>b) \leq I_{0}^{*}(b),
$$

for any given integer threshold $b \geq 0$.

We prove Theorem 1 by considering three types of events that lead to the delay-violation event $\{W(0)>b\}$ and computing their probabilities. We provide the proof in Appendix B.

Note that in [10], the authors derived another upper bound $\min \left\{(b+1) I_{X}, \min _{0 \leq c \leq b}\left\{I_{A}^{+}(b-c)+c I_{X}\right\}\right\}$, where $I_{A}^{+}(x) \triangleq \inf _{t>0} I_{A}^{+}(t, x)$ and $I_{A}^{+}(t, x) \triangleq \lim _{y \rightarrow x^{+}} I_{A}(t, y)$. We would like to remark that their upper bound was derived by considering two types of events that lead to the delay-violation event, which yet accounts for only a proper subset of the events that we consider in Appendix B Hence, their upper bound could be larger than $I_{0}^{*}(b)$ in some cases.

\section{SufFicient Conditions}

In [10], [11], the authors proposed the DWM policy and studied its rate-function delay optimality 2 ] (without the second part of Assumption 2) in some cases. Specifically, in [10], [11], the authors proved

\footnotetext{
${ }^{2}$ Although the delay metric considered in [10], [11] is slightly different from ours, both metrics are closely related. Moreover, the rate-function delay analysis for DWM in [10], [11] is also applicable for our defined rate-function as in (2).
} 
that DWM attains a rate-function that is no smaller than $\min \left\{(b+1) I_{X}, \min _{0 \leq c \leq b}\left\{I_{A}(b-c)+c I_{X}\right\}\right\}$, where $I_{A}(x) \triangleq \inf _{t>0} I_{A}(t, x)$. This is proved by showing that the FBS policy (with a properly chosen operating parameter $h$ ) can attain this rate-function and DWM dominates FBS for all values of $h$ in a sample-path sense. As pointed out in [10, Section V.D], there may be a gap between the rate-function attained by DWM and the upper bound derived in [10], depending on the value of $b$ and the arrival process. More specifically, it can be shown that for given $b \geq 0$, if $I_{A}(b-c)=I_{A}^{+}(b-c)$ for all values of $c \in\{0, \ldots, b\}$ for the given arrival process, then both FBS and DWM are rate-function delay-optimal.

However, it is unclear whether the DWM policy is rate-function delay-optimal in general. Moreover, its high complexity $O\left(n^{5}\right)$ renders it impractical. Hence, the grand challenge is to find low-complexity scheduling policies that are both throughput-optimal and rate-function delay-optimal. To that end, in this section, we first characterize easy-to-verify sufficient conditions for rate-function delay optimality in the many-channel many-user asymptotic regime and for throughput optimality in non-asymptotic settings. We then develop two classes of policies, called the Oldest Packets First (OPF) policies and the Maximum Weight in the Fluid limit (MWF) polices, that satisfy the sufficient condition for rate-function delay optimality and throughput optimality, respectively.

As discussed in the introduction, our ultimate goal is to develop low-complexity hybrid policies that are both rate-function delay-optimal and throughput-optimal. However, it is unclear that, just because one policy is rate-function delay-optimal and another one is throughput-optimal, their combinations will necessarily yield the right hybrid policy that is optimal in terms of both throughput and delay. As we will discuss further at the beginning of Section $\mathrm{V}$, our carefully chosen sufficient conditions possess some special features that allow us to construct low-complexity hybrid policies that are both rate-function delay-optimal and throughput-optimal.

\section{A. Rate-function Delay Optimality}

We start by presenting the main result of this section in the following theorem, which provides a sufficient condition for scheduling policies to be rate-function delay-optimal.

Theorem 2: Under Assumptions 2 and 3 a scheduling policy $\mathbf{P}$ is rate-function delay-optimal if in any time-slot, policy $\mathbf{P}$ can serve the $k$ oldest packets in that time-slot for the largest possible value of $k \in\{1,2, \ldots, n\}$.

To prove Theorem 2, we will exploit a dominance property (Lemma 3) of the policies that satisfy the above sufficient condition. We provide the proof of Theorem 2 in Appendix $\mathrm{C}$, and give the intuition behind it as follows. First, it is easy to see that the First-come First-serve (FCFS) policy, which serves 
the oldest packets first, is (sample-path) delay-optimal in a single-queue single-server system. Also, it is not hard to see that for a multi-queue multi-server system with full connectivity, where all pairs of queue and server are connected, a policy that chooses to serve the oldest packets (over the whole system) first is delay-optimal. These motivate us to ask a natural and interesting question: if a policy chooses to serve the oldest packets first in a multi-queue multi-server system with time-varying and partial connectivity (as we consider in this paper), does it achieve rate-function delay optimality? Note that in such a system, at most $n$ packets can be served in each time-slot. Hence, if in each time-slot a policy can serve all the $n$ oldest packets in the system (as in the case with full connectivity), this policy should yield optimal delay performance. However, due to the random connectivity between queues and servers, no policy may be able to do so. Hence, we propose a class of policies that choose to serve the $k$ oldest packets for the largest possible value of $k$. In other words, for any $k \in\{1,2, \ldots, n\}$, if the $k$ oldest packets can be served by some scheduling policy, then our proposed policies will serve these $k$ packets too.

A similar, but less thorough, analysis was also carried out in [10], [11]. There, the authors proposed the Frame Based Scheduling (FBS) policy, which aims to serve the oldest packets in each time-slot and can be viewed as an approximation of FCFS policy. The FBS policy serves packets in units of frames. With a given positive integer $h$ as the operating parameter, each frame is constructed such that: 1) the difference of the arrival times of any two packets within a frame must be no greater than $h$; and 2) the total number of packets in each frame is no greater than $n_{0}=n-L h$. In each time-slot, the packets arrived at the beginning of this time-slot are filled into the last frame until any of the above two conditions are violated, in which case a new frame will be opened. In any time-slot, the FBS policy serves the HOL frame that contains the oldest (up to $n_{0}$ ) packets with high probability for large $n$. As discussed at the beginning of this section, it has been shown that the FBS policy with a properly chosen operating parameter $h$ is rate-function delay-optimal in some cases.

However, FBS may not be rate-function delay-optimal in some other cases. Specifically, consider i.i.d. Bernoulli arrivals with $L=1$. As pointed out in [10], the rate-function attained by the FBS policy is not optimal in this scenario. We provide the intuition as follows. Suppose there are a total of $n t$ packet arrivals to the system in an interval of $t$ time-slots. It is easy to see that FBS needs at least $t+1$ time-slots to completely serve these packets since at most $n-L h$ packets can be served by FBS in one time-slot. This could lead to a sub-optimal rate-function. To see this, consider the perfect-matching policy defined as follows. Let $\mathcal{Q}$ and $\mathcal{S}$ denote the set of queues and set of servers, respectively. In a time-slot $\tau$, let $\mathcal{C} \triangleq\left\{C_{i, j}(\tau): C_{i, j}(\tau)=1\right\}$ denote the set of edges between $\mathcal{Q}$ and $\mathcal{S}$. Clearly, $G[\mathcal{Q} \cup \mathcal{S}, \mathcal{C}]$ forms a bipartite graph. If a perfect matching can be found in the bipartite graph $G[\mathcal{Q} \cup \mathcal{S}, \mathcal{C}]$, then the servers 
are allocated to serve the oldest packets in the respective queues as determined by the perfect matching. Otherwise, none of servers will be allocated to the queues. It has been shown in [6] that in each time-slot, a perfect matching can be found with high probability for large $n$. Hence, in the case described above, the perfect-matching policy needs only $t$ time-slots to drain all these $n t$ packets with high probability for large $n$, while FBS is sub-optimal.

On the other hand, the perfect-matching policy does not perform well in many other cases due to the fact that it cannot serve more than one packet from each queue in a time-slot. For example, consider the case where there are $L$ packets existing in $Q_{1}$ and the other queues are all empty. FBS can drain these packets within one time-slot with high probability, yet the perfect-matching policy needs at least $L$ time-slots.

The above discussions suggest that if we can find a policy that dominates both the FBS policy and the perfect-matching policy, there is a hope that this policy may be able to achieve the optimal rate-function in general. We will show in Lemma 3 that a policy that satisfies the sufficient condition in Theorem 2 , indeed dominates both the FBS policy and the perfect-matching policy in a sample-path sense.

In order to state the dominance property of Lemma 3 below, we consider the following versions of the FBS policy and the perfect-matching policy. Suppose that packet $p$ is the $x_{p}$-th arrival to the queue $Q_{q(p)}$ in time-slot $t_{p}$. Then, we define the weight of the packet $p$ in time-slot $t$ as $\hat{w}(p)=$ $t-t_{p}+\frac{L+1-x_{p}}{L+1}+\frac{n+1-q(p)}{(L+1)(n+1)}$. For two packets $p_{1}$ and $p_{2}$, we say $p_{1}$ is older than $p_{2}$ if $\hat{w}\left(p_{1}\right)>\hat{w}\left(p_{2}\right)$. The above way of defining the weight ensures that among the packets that arrive at the same time, the priority is given to the packet that has an earlier order of arrival in each queue; and further, among the packets (in different queues) with the same order of arrival, the priority is given to the packet that arrives to the queue with a smaller index. For the FBS policy, we assume that the packets with a larger weight are filled to the frame with a higher priority when there are multiple packets arriving at the same time. While for the perfect-matching policy, we require that in time-slot $t$, the perfect-matching policy only serves packets with the largest value of $t-t_{p}+\frac{L+1-x_{p}}{L+1}$. Under this version of the perfect-matching policy, it is possible that a queue may not have any of its packets served even if a perfect-matching is found and a server is allocated to the queue. It should be noted that the above versions of the FBS policy and the perfect-matching policy are used for analysis only. Next, we present the dominance property in the following lemma.

Lemma 3: Consider the versions of the FBS policy and the perfect-matching policy described above. Suppose that policy $\mathbf{P}$ satisfies the sufficient condition in Theorem 2 , Then, for any given sample path, by the end of any time-slot $t$, policy $\mathbf{P}$ has served every packet that the FBS policy or the perfect-matching 
policy has served.

We prove Lemma 3 by contradiction, and provide the proof in Appendix D. Further, by using of this dominance property, and following a similar argument as in the rate-function delay analysis for FBS (Theorem 2 of [10]), we prove Theorem 2] Specifically, we consider all the sample paths that lead to the delay-violation event. There are different ways that the delay-violation event can occur, each of which has a corresponding rate-function for its probability of occurring. Large-deviations theory then tells us that the rate-function for delay violation is determined by the smallest rate-function among these possibilities (i.e., "rare events occur in the most likely way".) An outline of the proof for Theorem 2 is provided in Appendix [C]

Next, we define a class of OPF policies as follows.

Definition 1: A scheduling policy $\mathbf{P}$ is said to be in the class of Oldest Packets First (OPF) policies if policy $\mathbf{P}$ satisfies the sufficient condition in Theorem 2

Clearly, the class of OPF policies are all rate-function delay-optimal. We would like to emphasize that the sufficient condition in Theorem 2 is very easy to verify and can be readily used to design other rate-function delay-optimal policies. Specifically, Theorem 2 enables us to identify a new ratefunction delay-optimal policy, called the DWM- $n$ policy, which substantially reduces the complexity to $O\left(n^{2.5} \log n\right)$. This in turn allows us to design low-complexity hybrid scheduling policies that are both throughput-optimal and rate-function delay-optimal (in Section V).

Now, we review the Delay Weighted Matching (DWM) policy proposed in [10], [11]. DWM operates in the following way. In each time-slot $t$, define the weight of the $l$-th packet of $Q_{i}$ as $Z_{i, l}(t)$, i.e., the delay of this packet at the beginning of time-slot $t$, which is measured since the time when this packet arrived to queue $Q_{i}$ until time-slot $t$. Then, construct a bipartite graph $G[X \cup Y, E]$ such that the vertices in $X$ correspond to the $n$ oldest packets from each of the $n$ queues and $Y$ is the set of all servers. Thus, $|X|=n^{2}$ and $|Y|=n$. Let $X_{i} \subseteq X$ be the set of packets from queue $Q_{i}$. If queue $Q_{i}$ is connected to server $S_{j}$, then for each packet $x \in X_{i}$, there is an edge between $x$ and $S_{j}$ in graph $G$ and the weight of this edge is set to the weight of packet $x$. The schedule is then determined by a maximum-weight matching over $G$. Clearly, DWM maximizes the sum of the delays of the packets scheduled.

It has been shown in [10], [11] that the DWM policy is rate-function delay-optimal in some cases. However, it is unclear whether it is delay-optimal in general. We would like to highlight that our proposed sufficient condition in Theorem 2 allows us to show that a slightly modified version of the DWM policy is rate-function delay-optimal in general (under an additional mild assumption - the second part of Assumption 2). Specifically, in the modified version of the DWM policy, we assign the weight of a 
packet $p$ as $\hat{w}(p)$ instead of its delay only. Then, by simply duplicating the proof of Lemma 7 in [10], we can show that the modified version of the DWM policy is an OPF policy and is thus rate-function delay-optimal.

However, the DWM policy still suffers from a high complexity, which renders it impractical. Specifically, DWM has a complexity of $O\left(n^{5}\right)$, since the complexity of finding a maximum-weight matching [14] over a bipartite graph $G[V, E]$ is $O\left(|V||E|+|V|^{2} \log |V|\right)$ in general, and the bipartite graph constructed by DWM has $|V|=O\left(n^{2}\right)$ and $|E|=O\left(n^{3}\right)$.

To overcome the high-complexity issue, we develop a simpler policy that is also in the class of the OPF policies (and is thus rate-function delay-optimal), but has a much lower complexity of $O\left(n^{2.5} \log n\right)$. The new policy is called the DWM- $n$ policy due to the high-level similarity with DWM. However, it exhibits critical differences when picking packets to construct the bipartite graph $G[X \cup Y, E]$ and finding the maximum-weight matching over $G$. The differences are as follows:

1) In each time-slot, instead of considering the $n$ oldest packets from each queue (and thus $n^{2}$ packets in total) as in DWM, DWM- $n$ considers only the $n$ oldest packets in the whole system. Hence, the bipartite graph constructed by DWM- $n$ has $|X|=n$ and $|Y|=n$.

2) The rest of the operations of DWM- $n$ are similar to that of DWM, i.e., the schedule is determined by a maximum-weight matching over $G$, except that DWM- $n$ finds a maximum-weight matching based on the vertex weights. Such a maximum-weight matching is also called Maximum Vertexweighted Matching (MVM) [15], [16]. Specifically, the weight of each vertex $p \in X$ is set to $\hat{w}(p)$ (i.e., the weight of the corresponding packet $p$ ), and the weight of each vertex in the set $Y$ is set to 0 .

In the following proposition, we show that the DWM- $n$ policy is rate-function delay-optimal and has a low complexity.

Proposition 4: The DWM-n policy is an OPF policy, and is thus rate-function delay-optimal under Assumptions 2 and 3 Further, the DWM- $n$ policy has a low complexity of $O\left(n^{2.5} \log n\right)$.

We provide the proof in Appendix E The fact that the DWM- $n$ policy is an OPF policy follows from a property of MVM [15] that if there exists a matching that matches all of the $k$ heaviest vertices, then any MVM matches all of the $k$ heaviest vertices as well. The low complexity of DWM- $n$ follows immediately from the fact that DWM- $n$ reduces the number of packets under consideration ( $n$ packets in total), and that an MVM in an $n \times n$ bipartite graph can be found in $O\left(n^{2.5} \log n\right)$ time [15]. Note that even if the DWM policy adopts MVM when determining the schedule, its complexity can only be reduced to $O\left(n^{4} \log n\right)$. 
Although the DWM- $n$ policy achieves rate-function delay optimality with a low complexity, it may not be throughput-optimal in general. This is because the DWM- $n$ policy considers only the $n$ oldest packets in the system. It is likely that certain servers may not be connected to any of the queues that contain these $n$ packets, which results in the server being idle and is thus a waste of service. Hence, DWM- $n$ is a lazy policy. In fact, we can construct a simple counter-example to show that the DWM- $n$ policy is, in general, not throughput-optimal as stated in Proposition 5

Proposition 5: The DWM-n policy is not throughput-optimal in general.

We prove Proposition 5 by constructing a special arrival pattern that forces certain servers to be idle, even when they can serve some of the queues. We provide the proof in Appendix $\mathrm{F}$ Proposition 5 suggests that a rate-function delay-optimal policy may not have good throughput performance (for a fixed $n$ ). This may appear counter-intuitive at the first glance. However, it should be noted that the rate-function delay optimality is studied in the asymptotic regime, i.e., when $n$ goes to infinity. Although the convergence of the rate-function is typically fast, the throughput performance may be poor for small to moderate values of $n$. Our simulation results (Fig. 3 in Section VI) will provide further evidence of this.

\section{B. Throughput Optimality}

In this section, we present a sufficient condition for throughput optimality in very general nonasymptotic settings.

Recall that $Q_{i}(t)$ denotes the length of queue $Q_{i}$ at the beginning of time-slot $t$ immediately after packet arrivals, $Z_{i, l}(t)$ denotes the delay of the $l$-th packet of $Q_{i}$ at the beginning of time-slot $t, W_{i}(t)=Z_{i, 1}(t)$ denotes the HOL packet delay of $Q_{i}$ at the beginning of time-slot $t$, and $C_{i, j}(t)$ denotes the connectivity between $Q_{i}$ and $S_{j}$ in time-slot $t$. Let $\mathcal{S}_{j}(t)$ denote the set of queues being connected to server $S_{j}$ in time-slot $t$, i.e., $\mathcal{S}_{j}(t)=\left\{1 \leq i \leq n \mid C_{i, j}(t)=1\right\}$, and let $\Gamma_{j}(t)$ denote the subset of queues in $\mathcal{S}_{j}(t)$ that have the largest weight in time-slot $t$, i.e., $\Gamma_{j}(t) \triangleq\left\{i \in \mathcal{S}_{j}(t) \mid W_{i}(t)=\max _{l \in \mathcal{S}_{j}(t)} W_{l}(t)\right\}$. We now present the main result of this section.

Theorem 6: Let $i(j, t)$ be the index of the queue that is served by server $S_{j}$ in time-slot $t$, under a scheduling policy $\mathbf{P}$. Under Assumption 1, policy $\mathbf{P}$ is throughput-optimal if there exists a constant $M>0$ such that, in any time-slot $t$ and for all $j \in\{1,2, \ldots, n\}$, queue $Q_{i(j, t)}$ satisfies that $W_{i(j, t)}(t) \geq Z_{r, M}(t)$ for all $r \in \Gamma_{j}(t)$ such that $Q_{r}(t) \geq M$.

We prove Theorem 6 using fluid limit techniques [13], [17], and provide the proof in Appendix G] The condition in Theorem 6 means the following: In each time-slot, each server chooses to serve a queue with HOL packet delay no less than the delay of the $M$-th packet in the queue with the largest HOL 
delay (among the queues connected to the server); if this queue (with the largest HOL delay) has less than $M$ packets, then the server may choose to serve any queue.

It is well-known that the MaxWeight Scheduling (MWS) policy [12], [13], [18]-[21] that maximizes the weighted sum of the rates, where the weights are queue lengths or delays, is throughput-optimal in very general settings, including the multi-channel system that we consider in this paper. The intuition behind Theorem 6 is that to achieve throughput optimality in our multi-channel system, it is sufficient for each server to choose a connected queue with a large enough weight such that this queue has the largest weight in the fluid limit. This relaxes the condition that each server has to find a queue with the largest weight in the original system, and thus significantly expands the set of known throughput-optimal policies.

Next, we define the class of Maximum Weight in the Fluid limit (MWF) policies as follows.

Definition 2: A policy $\mathbf{P}$ is said to be in the class of Maximum Weight in the Fluid limit (MWF) policies if policy $\mathbf{P}$ satisfies the sufficient condition in Theorem 6

Clearly, the class of MWF policies are all throughput-optimal. It is claimed in [10], [11] that the DWM policy is throughput-optimal, yet the throughput optimality was not explicitly proved there. For completeness, we state the following proposition on throughput optimality of the DWM policy, and provide its proof in Appendix $\mathrm{H}$

Proposition 7: The DWM policy is an MWF policy, and is thus throughput-optimal under Assumption 1

Next, we study a simple extension of the delay-based MaxWeight policy [12], [13], [21] that is throughput-optimal in our multi-channel system.

Delay-based MaxWeight Scheduling (D-MWS) policy: In each time-slot $t$, the scheduler allocates each server $S_{j}$ to serve queue $Q_{i(j, t)}$ such that $i(j, t)=\min \left\{i \mid i \in \Gamma_{j}(t)\right\}$. In other words, each server chooses to serve a queue that has the largest HOL delay (among all the queues connected to this server), breaking ties by picking the one with the smallest index if there are multiple such queues.

It is easy to see that D-MWS is an MWF policy and is thus throughput-optimal. Also, it is worth noting that D-MWS has a low complexity of $O\left(n^{2}\right)$ in our mutli-channel system. However, we can show that D-MWS suffers from poor delay performance. Specifically, we show in the following proposition that under D-MWS, the probability that the largest HOL delay exceeds any fixed threshold, is at least a constant, even if $n$ is large. This results in a zero rate-function.

Proposition 8: Consider i.i.d. Bernoulli arrivals, i.e., in each time-slot, and for each user, there is a packet arrival with probability $p$, and no arrivals otherwise. By allocating servers to queues according to 
D-MWS, we have that

$$
\limsup _{n \rightarrow \infty} \frac{-1}{n} \log \mathbb{P}(W(0)>b)=0
$$

for any fixed integer $b \geq 0$.

We provide the proof in Appendix $\square$ and explain the intuition behind it in the following. Note that under D-MWS, each server chooses to serve a connected queue having the largest weight without accounting for the decisions of the other servers. This way of allocating servers may incur an unbalanced schedule such that in each time-slot, with high probability, only a small fraction of the queues $(O(\log n)$ out of $n$ queues) get served, while the number of queues having arrivals is much larger $(O(n))$. This then leads to poor delay performance. By an argument similar to that in Theorem 3 of [7] (where the authors show that the Queue-length-based MaxWeight Scheduling (Q-MWS) policy results in a zero queue-length rate-function), we can show that under D-MWS, the delay-violation event occurs with at least a constant probability for any fixed threshold even if $n$ is large.

We conclude this section with a summary of the scheduling policies proposed and/or discussed in this section. The FBS policy is a good policy that is useful for the rate-function delay analysis of other policies, yet it is neither throughput-optimal nor rate-function delay-optimal in general. Although (the modified version of) the DWM policy is both throughput-optimal and rate-function delay-optimal, it yields an impractically high complexity. Our analysis shows that our proposed the DWM- $n$ policy is rate-function delay-optimal and substantially reduces the complexity to $O\left(n^{2.5} \log n\right)$, but it is not throughput-optimal. Further, we show that a simple throughput-optimal policy, the D-MWS policy, suffers from a zero ratefunction.

\section{HYBRID POLICIES}

It is clear from the previous section that a policy that satisfies the sufficient conditions in Theorems 2 and 6 is both throughput-optimal and rate-function delay-optimal. It remains however to find such a policy with a low complexity. Interestingly, our carefully chosen sufficient conditions possess the following special features, which allow us to construct a low-complexity hybrid policy that is both rate-function delay-optimal and throughput-optimal:

- The sufficient condition for throughput optimality has a decoupling feature, in the sense that the condition can be separately verified for each individual server.

- The sufficient condition for rate-function delay optimality guarantees not only rate-function delay optimality itself, but also that all scheduled servers for the $n$ oldest packets satisfy the sufficient condition for throughput optimality. 
Hence, by exploiting the above useful features of our sufficient conditions, we can now develop a class of two-stage hybrid OPF-MWF policies that runs an OPF policy (focusing on the $n$ oldest packets only) in stage 1, and runs an MWF policy in stage 2 over the remaining servers (that are not allocated in stage 1) only. We will then show that all policies in this class of hybrid OPF-MWF policies are both rate-function delay-optimal and throughput-optimal. In particular, we can find simple OPF-MWF policies with a low complexity $O\left(n^{2.5} \log n\right)$.

We now formally define the class of two-stage hybrid OPF-MWF policies.

Definition 3: A scheduling policy $\mathbf{P}$ is said to be in the class of hybrid OPF-MWF policies, if the following conditions are satisfied under policy $\mathbf{P}$ : In each time-slot $t$, there are two stages:

1) in stage 1 , it runs an OPF policy over the $n$ oldest packets only;

2 ) in stage 2, let $R(t)$ denote the set of servers that are not allocated by the OPF policy in stage 1 , and let $i(j, t)$ be the index of the queue that is matched by server $S_{j}$ for $j \in R(t)$ in stage 2 . There exists a constant $M>0$ such that in any time-slot $t$ and for all $j \in R(t)$, queue $Q_{i(j, t)}$ satisfies that $W_{i(j, t)}(t) \geq Z_{r, M}(t)$ for all $r \in \Gamma_{j}(t)$ such that $Q_{r}(t) \geq M$. In other words, it runs an MWF policy over the system with the remaining servers and packets.

In the following theorem, we show that the class of OPF-MWF policies are both rate-function delayoptimal and throughput-optimal.

Theorem 9: Any hybrid OPF-MWF policy is rate-function delay-optimal under Assumptions 2 and 3. and is throughput-optimal under Assumption 1

We provide the proof in Appendix \ and give the intuition behind it as follows. In stage 1, an OPF policy not only guarantees rate-function delay optimality, but also satisfies the sufficient condition for throughput optimality for all allocated servers in this stage. Note that the allocated servers and packets in stage 1 will not be considered in stage 2. In stage 2, we run an MWF policy for the remaining servers and packets only. Hence, it ensures that the sufficient condition for throughput optimality is satisfied for the remaining servers as well. Since the allocated servers and packets in stage 1 are not touched in stage 2, the satisfaction of the sufficient condition for delay optimality is not perturbed, and the sufficient condition for throughput optimality is also satisfied.

We note that the idea of combining different policies into (heuristic) hybrid policies to improve the overall performance, is not new. However, our goal in this paper is to achieve provable optimality in terms of both throughput and delay. Hence, the task of designing the right hybrid policy becomes much more challenging. Further, it is not necessary that all combinations of the OPF and MWF policies lead to desired hybrid policies. For example, it is unclear that the sufficient condition for throughput optimality 
can be satisfied if instead, we run an MWF policy in stage 1 and do post-processing by applying an OPF policy in stage 2. In this case, because the servers allocated by an MWF policy in stage 1 can be reallocated in stage 2 , the sufficient condition for throughput optimality may not hold any more. In contrast, our solutions exploit the special features of our carefully chosen sufficient conditions, and intelligently combine different policies in a right way, to achieve the optimal performance for both throughput and delay.

There are still many policies in the class of hybrid OPF-MWF policies. In the following, as an example, we show that the DWM- $n$ policy combined with the D-MWS policy yields an $O\left(n^{2.5} \log n\right)$-complexity hybrid OPF-MWF policy that is both throughput-optimal and rate-function delay-optimal. Let this policy be called DWM- $n$-MWS policy. Then, we present the main result of this paper in the following theorem.

Theorem 10: DWM-n-MWS policy is in the class of hybrid OPF-MWF policies, and is thus both throughput-optimal and rate-function delay-optimal. Further, DWM- $n$-MWS policy has a complexity of $O\left(n^{2.5} \log n\right)$.

To show that DWM- $n$-MWS is a hybrid OPF-MWF policy, it suffices to show that Condition 2) of Definition 3 is satisfied. We provide the proof in Appendix $\mathbb{K}$

\section{Vi. Simulation Results}

In this section, we conduct simulations to compare the performance of the scheduling policies proposed or discussed in this paper, where the Hybrid policy we consider is DWM- $n$-MWS policy. We also compare the delay performance of our proposed policies along with two $O\left(n^{2}\right)$-complexity queue-lengthbased policies (i.e., using queue lengths instead of delays to calculate weights when making scheduling decisions): Queue-based Server-Side-Greedy (Q-SSG) and Q-MWS, which have been studied in [6], [7]. We implement and simulate these policies in Java, and compare the empirical probabilities that the largest HOL delay in the system in any given time-slot exceeds a constant $b$, i.e., $\mathbb{P}(W(0)>b)$.

For the arrival processes, we consider bursty arrivals that are driven by a two-state Markov chain and are thus correlated over time. (We obtained similar results for i.i.d. arrivals over time, but omit them here due to space constraints.) We adopt the same parameter settings as in [10], [11]. For each user, there are 5 packet-arrivals when the Markov chain is in state 1, and no arrivals when the Markov chain is in state 2. The transition probability of the Markov chain is given by the matrix $[0.5,0.5 ; 0.1,0.9]$, and the state transitions occur at the end of each time-slot. The arrivals for each user are correlated over time, but they are independent across users. For the channel model, we first assume i.i.d. ON-OFF channels (as in Assumption 4) and set $q=0.75$, and later consider more general scenarios with heterogeneous users and 


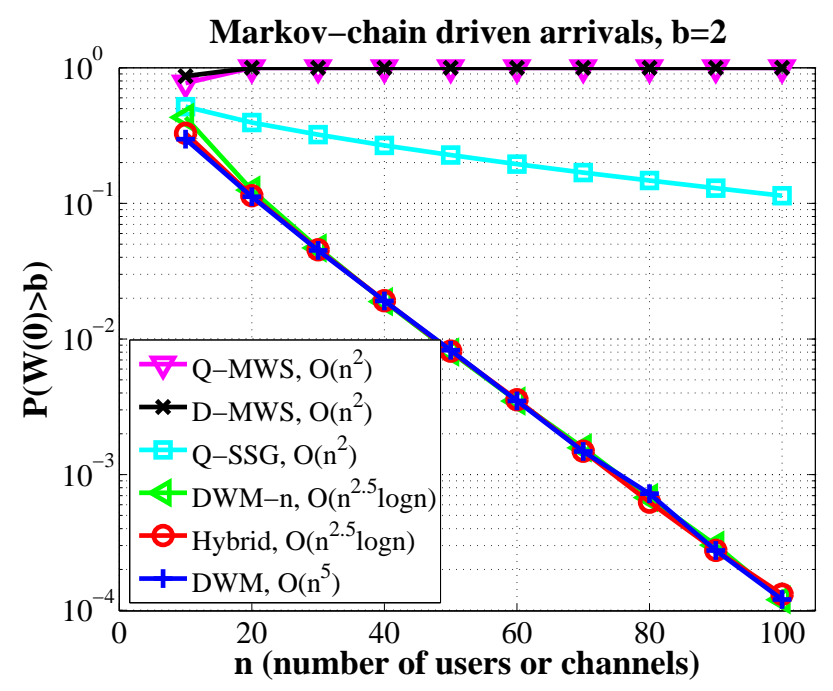

Fig. 2. Performance comparison of different scheduling policies in the case with homogeneous i.i.d. channels, for delay threshold $b=2$.

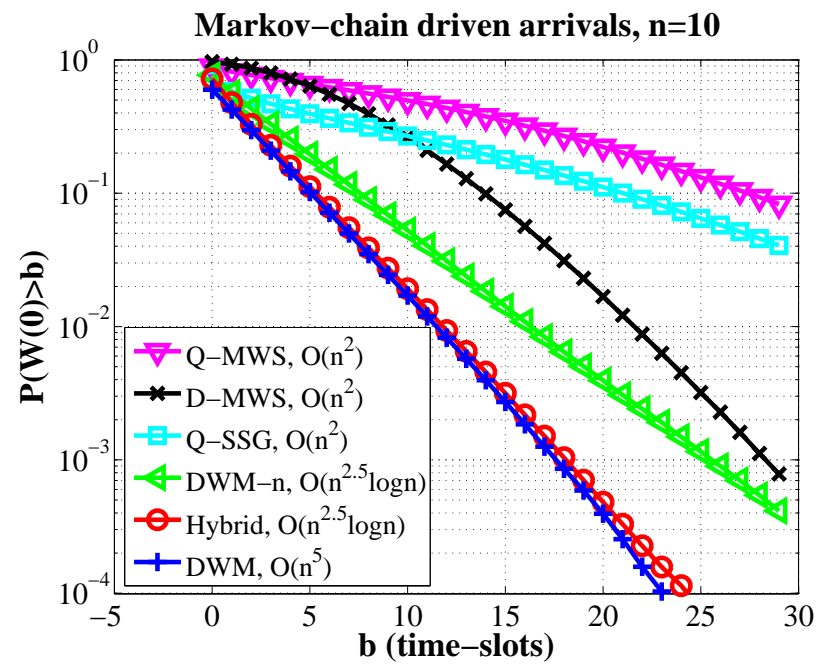

Fig. 3. Performance comparison of different scheduling policies in the case with homogeneous i.i.d. channels, for $n=10$ channels/users.

bursty channels that are correlated over time. We run simulations for a system with $n \in\{10,20, \ldots, 100\}$. The simulation period lasts for $10^{7}$ time-slots for each policy and each system.

The results are summarized in Figs. 2 and 3, where the complexity of each policy is labeled. In order to compare the rate-function $I(b)$ as defined in Eq. (2), we plot the probability over the number of channels or users, i.e., $n$, for a fixed value of threshold $b$. In Fig. 2 , we compare the rate-function $I(b)$ 


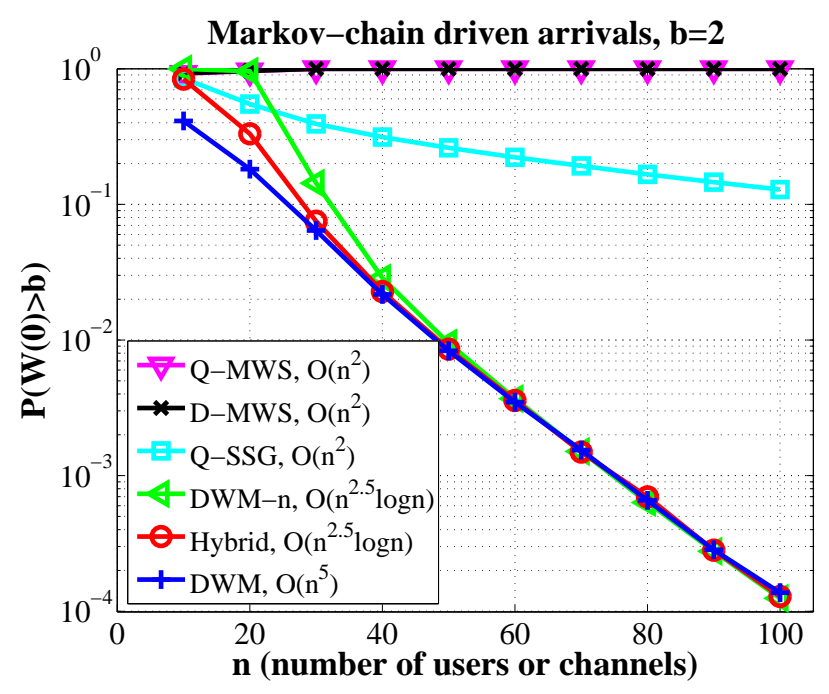

Fig. 4. Performance comparison of different scheduling policies in the case with Markov-chain driven heterogeneous channels, for delay threshold $b=2$.

of different scheduling policies for $b=2$. The negative of the slope of each curve can be viewed as the rate-function for the corresponding policy. From Fig. 2, we observe that the Hybrid and DWM- $n$ policies perform closely to DWM, and that D-MWS and Q-MWS have a zero rate-function, which supports our analytical results. Further, the results show that the delay-based policies (DWM, DWM- $n$ and Hybrid) consistently outperform Q-SSG in terms of delay performance, despite that it has been shown through simulations that Q-SSG performs closely to a rate-function (queue-length) optimal policy [6], [7]. This provides further evidence of the fact that good queue-length performance does not necessarily translate to good delay performance.

We also plot the probability over delay threshold $b$ as in [6]-[8], [10], [11] to investigate the performance of different policies when $n$ is small. In Fig. 3, we report the results for $n=10$ and $b \in\{0,1,2, \ldots, 29\}$. From Fig. 3, we observe that the Hybrid policy consistently performs closely to DWM for almost all values of $b$ that we consider, while DWM- $n$ is worse than DWM. This is because DWM- $n$ may not schedule all the servers, and the probability that some of the servers are kept idle can be significant when $n$ is small.

Finally, we evaluate the performance of different scheduling policies in more realistic scenarios, where users are heterogeneous and channels are correlated over time. Specifically, we consider channels that can be modeled by a two-state Markov chain, where the channel is "ON" when the Markov chain is in state 1, and is "OFF" when the Markov chain is in state 2. This type of channel model can be viewed as a special 
case of the Gilbert Elliot model that is widely used for describing bursty channels. We assume that there are two classes of users: users with an odd index are called near-users, and users with an even index are called far-users. Different classes of users see different channel conditions: near-users see better channel condition, and far-users see worse channel condition. We assume that the transition probability matrices of channels for near-users and far-users are $[0.833,0.167 ; 0.5,0.5]$ and $[0.5,0.5 ; 0.167,0.833]$, respectively. The arrival processes are assumed to be the same as in the previous case. Also, the delay requirements are assumed to be the same for different classes of users, i.e., we still consider the probability that the largest HOL delay exceeds a fixed threshold, without distinguishing different classes of users.

The results are summarized in Fig. 4. We observe similar results as in the previous case, where channels are i.i.d. in time. In particular, our low-complexity policies (DWM- $n$ and Hybrid) again perform closely to DWM, in terms of rate-function, although the delay-violation probability is a bit smaller under DWM when $n$ is not large (i.e., $n<50$ ), which is expected. Note that in this scenario, rate-function delayoptimal policies are not known yet. For future work, it would be interesting to explore whether our proposed policies can achieve optimality of both throughput and delay in more general scenarios.

\section{CONCLUSION}

In this paper, we addressed the question of designing low-complexity scheduling policies that provide optimal performance of both throughput and delay in multi-channel systems. We derived simple and easy-to-verify sufficient conditions for throughput optimality and rate-function delay optimality, which allowed us to later develop a class of low-complexity hybrid policies that simultaneously achieve both throughput optimality and rate-function delay optimality.

Our work in this paper leads to many interesting questions that are worth exploring in the future. It would be interesting to know if one can further relax the sufficient conditions, and design even

simpler policies that can provide optimal performance for both throughput and delay. Further, it would be worthwhile to analytically characterize the fundamental trade-off between performance and complexity.

Further, it is important to investigate the scheduling problem in more realistic scenarios, e.g., accounting for more general multi-rate channels that are correlated over time, rather than i.i.d. ON-OFF channels, and heterogeneous users with different statistics as well as different delay requirements. Our hope is to find efficient schedulers that can guarantee a nontrivial lower bound of the optimal rate-function, if it is too hard to achieve (or prove) the optimal delay performance itself in more general scenarios.

Finally, it is interesting and important for us to understand the delay performance beyond rate-function optimality as we considered in this paper. The log-asymptotic results from the large-deviations analysis 
may not suffice, since they do not account for the pre-factor of the delay-violation probability. Therefore, a very important direction is to analyze and understand the exact delay asymptotics as well as the mean delay performance.

\section{APPENDIX A}

\section{The Optimal Throughrut Region $\Lambda^{*}$}

We can characterize the optimal throughput region $\Lambda^{*}$ of our multi-channel systems in a similar manner to that of single-channel systems in [13].

We start with discussions for a single-channel system with $n$ users in a more general setting. Specifically, suppose that there is a finite set $\mathcal{M}=\{1,2, \ldots,|\mathcal{M}|\}$ of global server states (where the server state accounts for the state of the links between the server and all users). For each state $m \in \mathcal{M}$, there is an associated service rate vector $r^{m}=\left[r_{i}^{m}, 1 \leq i \leq n\right]$, where $r_{i}^{m}$ is the maximum number of packets that can be transmitted to $Q_{i}$ when the server is in state $m$ (under Assumption 4 of ON-OFF channels, we have $r_{i}^{m} \in\{0,1\}$ for all $m$ and $i$ ). We assume that the random channel state process is a stationary and ergodic discrete-time Markov chain within the state space $\mathcal{M}$. We let $\pi=\left[\pi_{m}, m \in \mathcal{M}\right]$ denote the stationary distribution of this Markov chain, where $\pi_{m}>0$ for all $m \in \mathcal{M}$.

As in [13], consider a Static Service Split (SSS) policy, associated with an $|\mathcal{M}| \times n$ stochastic matrix $\phi=\left[\phi_{m, i}, m \in \mathcal{M}, 1 \leq i \leq n\right]$, where $\phi_{m, i} \geq 0$ for all $m$ and $i$, and $\sum_{1 \leq i \leq n} \phi_{m, i}=1$ for every $m$. Under the SSS policy, the server chooses to serve $Q_{i}$ with probability $\phi_{m, i}$ when the server is in state $m$. Clearly, the (long-term average) service rate vector can be represented by $\nu=\left[\nu_{i}, 1 \leq i \leq n\right]=\nu(\phi)$, where $\nu_{i}=\sum_{m \in \mathcal{M}} \pi_{m} \phi_{m, i} r_{i}^{m}$. Then, the set of all feasible (long-term average) service rate vector can be represented as

$$
\mathcal{R}=\{\nu \mid \nu=\nu(\phi) \text { for some stochastic matrices } \phi\} .
$$

Hence, the optimal throughput region can be represented as

$$
\Lambda^{*}=\{\lambda \mid \lambda \leq \nu \text { for some vector } \nu \in \mathcal{R}\}
$$

Now, consider a multi-channel system with $n$ orthogonal channels. Let $\mu_{i, j}$ denote the feasible (longterm average) service rate that can be allocated to queue $Q_{i}$ from server $S_{j}$, and let the vector $\mu_{j}=$ $\left[\mu_{i, j}, 1 \leq i \leq n\right]$ denote a feasible service rate allocation by server $S_{j}$. For each server $S_{j}$, the set of all such feasible vectors $\mu_{j}$ is denoted by $\mathcal{R}_{j}$. Note that the characterization of $\mathcal{R}_{j}$ has already accounted for the time-varying channel-states. Let $\mu=\left[\mu_{j}, 1 \leq j \leq n\right]$ denote a feasible service rate matrix, and the 
set of all such feasible matrices $\mu$ can be represented as $\mathcal{R}=\mathcal{R}_{1} \times \mathcal{R}_{2} \times \cdots \times \mathcal{R}_{n}$. Hence, the optimal throughput region $\Lambda^{*}$ can be represented as

$$
\Lambda^{*}=\left\{\lambda \mid \lambda_{i} \leq \sum_{j=1}^{n} \mu_{i, j} \text { for all } i, \text { for some matrix } \mu \in \mathcal{R}\right\} .
$$

Note that our multi-channel system under Assumption 4 of ON-OFF channel model is a special case of the above scenario.

\section{APPENDIX B}

\section{ProOF OF THEOREM 1}

We begin with stating an important property of $I_{A}(t, x)$ in the following lemma, which will be used in deriving the upper bound in Theorem 11 Recall that we define the quantity $I_{A}^{+}(t, x) \triangleq \lim _{y \rightarrow x^{+}} I_{A}(t, y)$.

Lemma 11: Suppose $L>1$. For any given integer $t>0$, and for all $x \in[0,(L-1) t)$, the limit $I_{A}^{+}(t, x)=\lim _{y \rightarrow x^{+}} I_{A}(t, y)$ exists and we have $I_{A}(t, x)=I_{A}^{+}(t, x)$.

Proof: Consider any given integer $t>0$. First, note that the total number of packet arrivals to the system during an interval of $t$ time-slots cannot exceed $L n t$. Hence, we only need to consider $I_{A}(t, x)$ defined on $[0,(L-1) t]$. By the second part of Assumption 2 it is easy to see that $I_{A}(t, x)$ must be finite in $[0,(L-1) t)$. Note that $I_{A}(t, x)$ is a supremum (over $\theta$ ) of linear functions (of $x$ ). Hence, $I_{A}(t, x)$ is a convex function (of $x$ ), and is thus continuous on $(0,(L-1) t)$ (i.e., the interior of $[0,(L-1) t]$ ) [22, Pg. 68]. Further, it is easy to see that $I_{A}(t, x)$ is monotone (non-decreasing) on $[0,(L-1) t]$ due to (3). Hence, it is not hard to show that $I_{A}(t, x)$ is right-continuous at the left-most point $x=0$. Therefore, the $\operatorname{limit}_{y \rightarrow x^{+}} I_{A}(t, y)$ exists and we have $I_{A}(t, x)=I_{A}^{+}(t, x)$ for any $x \in[0,(L-1) t)$.

First, we focus on the case where $L>1$, and consider three types of events, $\mathcal{E}_{1}, \mathcal{E}_{2}^{c}$, and $\mathcal{E}_{3}^{c}$, that imply the delay-violation event $\{W(0)>b\}$.

Event $\mathcal{E}_{1}$ : Suppose that there is a packet that arrives to the network in time-slot $-b-1$. Without loss of generality, we assume that the packet arrives to queue $Q_{1}$. Further, suppose that $Q_{1}$ is disconnected from all $n$ servers in all time-slots from $-b-1$ to -1 .

Then, at the beginning of time-slot 0 , this packet is still in the network and has a delay of $b+1$. This implies $\mathcal{E}_{1} \subseteq\{W(0)>b\}$. Note that the probability that event $\mathcal{E}_{1}$ occurs can be computed as

$$
\mathbb{P}\left(\mathcal{E}_{1}\right)=(1-q)^{n(b+1)}=e^{-n(b+1) I_{X}} .
$$

Hence, we have

$$
\mathbb{P}(W(0)>b) \geq e^{-n(b+1) I_{X}},
$$


and thus

$$
\limsup _{n \rightarrow \infty} \frac{-1}{n} \log \mathbb{P}(W(0)>b) \leq(b+1) I_{X} .
$$

Event $\mathcal{E}_{2}^{c}$ : Consider any fixed $c \in\{0,1, \ldots, b\}$ and any $t>t_{b-c}$. Recall that $t_{b-c}=\frac{b-c}{L-1}$. Then, for all $t>t_{b-c}$, we have $b-c<(L-1) t$, and thus $I_{A}(t, b-c)=I_{A}^{+}(t, b-c)$ from Lemma 11. Hence, for any fixed $\epsilon>0$, there exists a $\delta>0$ such that $I_{A}(t, b-c+\delta) \leq I_{A}^{+}(t, b-c)+\epsilon=I_{A}(t, b-c)+\epsilon$. Suppose that from time-slot $-t-b$ to $-b-1$, the total number of packet arrivals to the system is greater than or equal to $n t+n(b-c+\delta)$, and let $p_{(b-c+\delta)}$ denote the probability that this event occurs. Then, from Cramer's Theorem, we have $\lim _{n \rightarrow \infty} \frac{-1}{n} \log p_{(b-c+\delta)}=I_{A}(t, b-c+\delta) \leq I_{A}(t, b-c)+\epsilon$. Clearly, the total number of packets that are served in any time-slot is no greater than $n$. For any fixed $\delta$, we have $n \delta \geq 1$ for large enough $n$ (when $n \geq \frac{1}{\delta}$ ). Hence, if the above event occurs, at the end of time-slot $-c-1$, the system contains at least one packet that arrived before time-slot $-b$. Without loss of generality, we assume that this packet is in $Q_{1}$. Now, assume that $Q_{1}$ is disconnected from all $n$ servers in the next $c$ time-slots, i.e., from time-slot $-c$ to -1 . This occurs with probability $(1-q)^{c n}=e^{-n c I_{X}}$, independently of all the past history. Hence, at the beginning of time-slot 0 , there is still a packet that arrived before time-slot $-b$. Thus, we have $W(0)>b$ in this case. This implies $\mathcal{E}_{2}^{c} \subseteq\{W(0)>b\}$. Note that the probability that event $\mathcal{E}_{2}^{c}$ occurs can be computed as

$$
\mathbb{P}\left(\mathcal{E}_{2}^{c}\right)=p_{(b-c+\delta)} e^{-n c I_{X}}
$$

Hence, we have

$$
\mathbb{P}(W(0)>b) \geq p_{(b-c+\delta)} e^{-n c I_{X}},
$$

and thus

$$
\limsup _{n \rightarrow \infty} \frac{-1}{n} \log \mathbb{P}(W(0)>b) \leq I_{A}(t, b-c)+\epsilon+c I_{X} .
$$

Since the above inequality holds for any $c \in\{0,1, \ldots, b\}$, any $t>t_{b-c}$, and any $\epsilon>0$, by letting $\epsilon$ tend to 0 , taking the infimum over all $t>t_{b-c}$, and taking the minimum over all $c \in\{0,1, \ldots, b\}$, we have

$$
\begin{aligned}
\limsup _{n \rightarrow \infty} & \frac{-1}{n} \log \mathbb{P}(W(0)>b) \\
& \leq \min _{c \in\{0,1, \ldots, b\}}\left\{\inf _{t>t_{b-c}} I_{A}(t, b-c)+c I_{X}\right\} .
\end{aligned}
$$

Event $\mathcal{E}_{3}^{c}$ : Consider any fixed $c \in \Psi_{b}$. Suppose that from time-slot $-t_{b-c}-b$ to $-b-1$, the total number of packet arrivals to the system is equal to $n t_{b-c}+n(b-c)=n L t_{b-c}$, and let $p_{(b-c)}^{\prime}$ denote the probability that this event occurs. Note that the total number of packet arrivals to the system from time-slot $-t_{b-c}-b$ 
to $-b-1$ can never exceed $n L t_{b-c}$. Then, from Cramer's Theorem, we have $\lim _{n \rightarrow \infty} \frac{-1}{n} \log p_{(b-c)}^{\prime}=$ $I_{A}\left(t_{b-c}, b-c\right)$. Clearly, the total number of packets that can be served during the interval $\left[-t_{b-c}-b,-c-1\right]$ is no greater than $n\left(t_{b-c}+b-c\right)=n L t_{b-c}$. Suppose that there exists one queue that is disconnected from all the servers in any one time-slot in the interval $\left[-t_{b-c}-b,-c-1\right]$. Then, at the end of timeslot $-c-1$, the system contains at least one packet that arrived before time-slot $-b$. Without loss of generality, we assume that queue $Q_{1}$ is disconnected from all the servers in a time-slot, say time-slot $-t_{b-c}-b$. This event occurs with probability $(1-q)^{n}=e^{-n I_{X}}$. Further, assume that $Q_{1}$ is disconnected from all the $n$ servers in the next $c$ time-slots, i.e., from time-slot $-c$ to -1 . This occurs with probability $(1-q)^{c n}=e^{-n c I_{X}}$, independently of all the past history. Hence, at the beginning of time-slot 0 , there is still a packet that arrived before time-slot $-b$. Thus, we have $W(0)>b$ in this case. This implies $\mathcal{E}_{3}^{c} \subseteq\{W(0)>b\}$. Note that the probability that event $\mathcal{E}_{3}^{c}$ occurs can be computed as

$$
\mathbb{P}\left(\mathcal{E}_{3}^{c}\right)=p_{(b-c)}^{\prime} e^{-n(c+1) I_{X}}
$$

Hence, we have

$$
\mathbb{P}(W(0)>b) \geq p_{(b-c)}^{\prime} e^{-n(c+1) I_{X}},
$$

and thus

$$
\limsup _{n \rightarrow \infty} \frac{-1}{n} \log \mathbb{P}(W(0)>b) \leq I_{A}\left(t_{b-c}, b-c\right)+(c+1) I_{X} .
$$

Since the above inequality holds for any $c \in \Psi_{b}$, by taking the minimum over all $c \in \Psi_{b}$, we have, for $L>1$

$$
\begin{aligned}
\limsup _{n \rightarrow \infty} & \frac{-1}{n} \log \mathbb{P}(W(0)>b) \\
& \leq \min _{c \in \Psi_{b}}\left\{I_{A}\left(t_{b-c}, b-c\right)+(c+1) I_{X}\right\} .
\end{aligned}
$$

Considering events $\mathcal{E}_{1}, \mathcal{E}_{2}^{c}$, and $\mathcal{E}_{3}^{c}$, we have

$$
\begin{aligned}
& \limsup _{n \rightarrow \infty} \frac{-1}{n} \log \mathbb{P}(W(0)>b) \\
& \leq \min \left\{(b+1) I_{X},\right. \\
& \min _{0 \leq c \leq b}\left\{\inf _{t>t_{b-c}} I_{A}(t, b-c)+c I_{X}\right\}, \\
&\left.\min _{c \in \Psi_{b}}\left\{I_{A}\left(t_{b-c}, b-c\right)+(c+1) I_{X}\right\}\right\} \\
&=I_{0}(b) .
\end{aligned}
$$

Next, we consider the case where $L=1$. In this case, we only need to consider event $\mathcal{E}_{1}$, and we have $\lim \sup _{n \rightarrow \infty} \frac{-1}{n} \log \mathbb{P}(W(0)>b) \leq(b+1) I_{X}$. 
Combining both cases of $L=1$ and $L>1$, we have $\lim _{\sup _{n \rightarrow \infty}} \frac{-1}{n} \log \mathbb{P}(W(0)>b) \leq I_{0}^{*}(b)$. This completes our proof.

\section{APPENDIX C}

\section{PROOF OF THEOREM 2}

Suppose policy $\mathbf{P}$ satisfies the sufficient condition in Theorem 2 . We want to show that for any given integer threshold $b \geq 0$, the rate-function attained by policy $\mathbf{P}$ is no smaller than $I_{0}^{*}(b)$. The proof follows a similar argument as in the proof of Theorem 2 in [10]. However, our proof exhibits the following key difference. In [10], the authors prove that the FBS policy can attain a certain rate-function, which, in some cases only, meets the upper bound derived in [10]. In contrast, in the following proof, by exploiting the dominance property over both the FBS policy and the perfect-matching policy in Lemma 3, we will show that the rate-function attained by policy $\mathbf{P}$ is always no smaller than the upper bound $I_{0}^{*}(b)$ that we derived in Theorem 1 and is thus optimal.

We first consider the case of $L>1$, and want to show that the rate-function attained by policy $\mathbf{P}$ is no smaller than $I_{0}(b)$.

In the following proof, we will use the dominance property of policy $\mathbf{P}$ over the FBS policy and the perfect-matching policy considered in Lemma 3 . We first choose the value of parameter $h$ for FBS based on the statistics of the arrival process. We fix $\delta<\frac{2}{3}$ and $\epsilon<\frac{p}{2}$. Then, from Assumption 3, there exists a positive function $I_{B}(\epsilon, \delta)$ such that for all $n \geq N_{B}(\epsilon, \delta)$ and $t \geq T_{B}(\epsilon, \delta)$, we have

$$
\mathbb{P}\left(\frac{\sum_{\tau=l+1}^{l+t} \mathbb{1}_{\{|A(\tau)-p n|>\epsilon n\}}}{t}>\delta\right)<\exp \left(-n t I_{B}(\epsilon, \delta)\right),
$$

for any integer $l$. We then choose

$$
h=\max \left\{T_{B}(\epsilon, \delta),\left\lceil\frac{1}{(p-\epsilon)\left(1-\frac{3 \delta}{2}\right)}\right\rceil,\left\lceil\frac{2 I_{0}(b)}{I_{B}(\epsilon, \delta)}\right\rceil\right\}+1 .
$$

The reason for choosing the above value of $h$ will become clear later on. Recall from Assumption 2 that $L$ is the maximum number of packets that can arrive to a queue in any time-slot $t$. Let $H=L h$. Then, $H$ is the maximum number of packets that can arrive to a queue during an interval of $h$ time-slots, and is thus the maximum number of packets from the same queue in a frame.

Let $L(-b)$ be the last time before time-slot $-b$, when the backlog is empty, i.e., all the queues have a queue-length of zero. Also, let $\mathcal{E}_{t}$ be the set of sample paths such that $L(-b)=-t-b-1$ and $W(0)>b$ under policy $\mathbf{P}$. Then, we have

$$
\mathbb{P}(W(0)>b)=\sum_{t=1}^{\infty} \mathbb{P}\left(\mathcal{E}_{t}\right)
$$


Let $\mathcal{E}_{t}^{F}$ and $\mathcal{E}_{t}^{P M}$ be the set of sample paths such that given $L(-b)=-t-b-1$, the event $W(0)>b$ occurs under the FBS policy and the perfect-matching policy, respectively. Recall that policy $\mathbf{P}$ dominates both the FBS policy and the perfect-matching policy. Then, for any $t>0$ we have

$$
\mathcal{E}_{t} \subseteq \mathcal{E}_{t}^{F} \cap \mathcal{E}_{t}^{P M}
$$

Recall that $p$ is the mean arrival rate to a queue. Now, we choose any fixed real number $\hat{p} \in(p, 1)$, and fix a finite time $t^{*}$ as

$$
t^{*} \triangleq \max \left\{T_{1},\left\lceil\frac{I_{0}(b)}{I_{B X}}\right\rceil, \max \left\{t_{b-c} \mid c \in \Psi_{b}\right\}\right\}
$$

where

$$
T_{1} \triangleq \max \left\{T_{B}\left(\hat{p}-p, \frac{1-\hat{p}}{6(L+2)}\right),\left\lceil\frac{6}{1-\hat{p}}\right\rceil\right\}
$$

and

$$
I_{B X} \triangleq \min \left\{\frac{(1-\hat{p}) I_{X}}{9}, I_{B}\left(\hat{p}-p, \frac{1-\hat{p}}{6(L+2)}\right)\right\} .
$$

The reason for defining the above value of $t^{*}$ will become clear later on. Then, we apply (7) to (6) and split the summation as

$$
\mathbb{P}(W(0)>b) \leq P_{1}+P_{2},
$$

where

$$
P_{1} \triangleq \sum_{t=1}^{t^{*}} \mathbb{P}\left(\mathcal{E}_{t}^{F} \cap \mathcal{E}_{t}^{P M}\right)
$$

and

$$
P_{2} \triangleq \sum_{t=t^{*}}^{\infty} \mathbb{P}\left(\mathcal{E}_{t}^{F} \cap \mathcal{E}_{t}^{P M}\right) .
$$

We divide the proof into two parts. In Part 1, we show that there exists a finite $N_{1}>0$ such that for all $n \geq N_{1}$, we have

$$
P_{1} \leq C_{1} n^{7 b H} e^{-n I_{0}(b)} .
$$

Then, in Part 2, we show that there exists a finite $N_{2}>0$ such that for all $n \geq N_{2}$, we have

$$
P_{2} \leq 4 e^{-n I_{0}(b)}
$$

Finally, combining both parts, we have

$$
\mathbb{P}(W(0)>b) \leq\left(C_{1} n^{7 b H}+4\right) e^{-n I_{0}(b)},
$$

for all $n \geq N \triangleq \max \left\{N_{1}, N_{2}\right\}$. By taking logarithm and limit as $n$ goes to infinity, we obtain $\liminf _{n \rightarrow \infty} \frac{-1}{n} \log \mathbb{P}(W(0)>b) \geq I_{0}(b)$, and thus the desired results. 
Before we prove Part 1 and Part 2, we derive the following properties of the FBS policy and the perfect-matching policy, which will be used in the proof.

We first calculate an upper bound on the probability that during interval $[-t-b,-1]$, there are exactly $t+a$ frames that can be served by the FBS policy, for some $a \leq b$. We define the random variable $X_{F}(t)=1$ if there exists a schedule such that a frame can be successfully served in time-slot $t$ under FBS, and $X_{F}(t)=0$, otherwise. Let $X_{F}(i, j)$ denote the total number of frames served by FBS from time-slot $i$ to $j$. Given any sample path in the set $\mathcal{E}_{t}$, i.e., $L(-b)=-t-b-1$ and $W(0)>b$, the backlog never becomes empty under policy $\mathbf{P}$ during the interval of $[-t-b,-1]$. Since policy $\mathbf{P}$ dominates the FBS policy, then the buffer is non-empty under the FBS policy during the interval of $[-t-b,-1]$. Hence, we have

$$
X_{F}(-t-b,-1)=\sum_{\tau=-t-b}^{-1} X_{F}(\tau) .
$$

From Lemma 6 of [10], there exists an $N_{F}>0$, such that for all $n \geq N_{F}$ the probability that there does not exist a schedule such that a frame can be served in each time-slot is no greater than $\left(\frac{n}{1-q}\right)^{7 H} e^{-n \log \frac{1}{1-q}}$. Hence, we have

$$
\begin{aligned}
& \mathbb{P}\left(\sum_{\tau=-t-b}^{-1} X_{F}(\tau)=t+a\right) \\
& \leq\left(\begin{array}{c}
t+b \\
t+a
\end{array}\right)\left(\frac{n}{1-q}\right)^{7 b H} e^{-(b-a) n \log \frac{1}{1-q}} \\
& \leq 2^{t+b}\left(\frac{n}{1-q}\right)^{7 b H} e^{-n(b-a) I_{X}} .
\end{aligned}
$$

Next, we define the random variable $X_{P M}(t)=1$ if a perfect-matching can be found in time-slot $t$, and $X_{P M}(t)=0$, otherwise. From Lemma 1 of [6], there exists an $N_{P M}>0$, such that for all $n \geq N_{P M}$ the probability that no perfect matching can be found in each time-slot is no greater than $3 n e^{-n \log \frac{1}{1-q}}$. Then, we can similarly show that

$$
\mathbb{P}\left(\sum_{\tau=-t-b}^{-1} X_{P M}(\tau)=t+a\right) \leq 2^{t+3 b} n^{b} e^{-n(b-a) I_{X}} .
$$

It is easy to observe that the right hand side of (12) and (13) is a monotonically increasing function in $a$.

Part 1: Consider any $t \in\left\{1,2, \ldots, t^{*}\right\}$. We let $\mathcal{E}_{t}^{\alpha}$ denote the set of sample paths in which there are at least $n$ packet arrivals to the system during every $h-1$ time-slots in the interval of $[-t-b,-b-1]$. Let $\mathcal{E}_{t}^{\beta}$ denote the set of sample paths in which $\frac{A(-t-b,-b-1)}{n_{0}}-\sum_{\tau=-t-b}^{-1} X_{F}(\tau)>0$ under the FBS policy. Since in any sample path of $\mathcal{E}_{t}$ the backlog never becomes empty (under policy $\mathbf{P}$ ) during the 
interval of $[-t-b,-b-1]$ and policy $\mathbf{P}$ dominates the FBS policy, then in any sample path of $\mathcal{E}_{t}^{\alpha}$ the backlog never becomes empty (under the FBS policy) during the interval of $[-t-b,-b-1]$. Similar as in the proof of Theorem 2 of [10], using Lemma 9 of [10], we can show that

$$
\mathcal{E}_{t}^{F} \subseteq\left(\mathcal{E}_{t}^{\alpha}\right)^{c} \cup \mathcal{E}_{t}^{\beta}
$$

and along with the choice of $h$ (as chosen earlier), we can show that there exist $N_{3}>0$ and $C_{2}>0$ such that for all $n \geq N_{3}$,

$$
\mathbb{P}\left(\mathcal{E}_{t}^{\alpha}\right)>1-C_{2} t e^{-n I_{0}(b)}
$$

Here, we do not duplicate the detailed proofs for (14) and (15), and refer the interested readers to [10] for details.

Next, we compute the probability of $\mathcal{E}_{t}^{\beta}$ for each $t$. For any fixed integer $t>0$, we derive an upper bound for the probability of a large burst of arrivals during an interval of $t$ time-slots. Let $\theta_{t} \triangleq \operatorname{argmax}_{\theta}[\theta(t+$ $\left.x)-\lambda_{A_{i}(-t+1,0)}(\theta)\right]$, and let $\theta^{*} \triangleq \max \left\{\theta_{1}, \theta_{2}, \ldots, \theta_{t^{*}}\right\}$. Recall that $n_{0}=n-H$. We know from the Chernoff bound that for any $x \in[0,(L-1) t]$,

$$
\begin{aligned}
& \mathbb{P}\left(A(-t+1,0)>n_{0}(t+x)\right) \\
& =\mathbb{P}(A(-t+1,0) \geq(n-H)(t+x)+1) \\
& \leq e^{-n\left(\theta_{t}(t+x)-\lambda_{A_{i}(-t+1,0)}\left(\theta_{t}\right)\right)+(H(t+x)-1) \theta_{t}} \\
& \leq e^{-n I_{A}(t, x)} e^{(H(t+x)-1) \theta^{*}} .
\end{aligned}
$$

Recall that $t_{x}=\frac{x}{L-1}$. We first consider any $t \in\left\{1,2, \ldots, t^{*}\right\} \backslash\left\{t_{b-c^{\prime}} \mid c^{\prime} \in \Psi_{b}\right\}$. For these values of $t$, we will use the dominance property over FBS, i.e., $\mathcal{E}_{t} \subseteq \mathcal{E}_{t}^{F}$. Let $c_{t}$ be such that $t_{b-c_{t}}<t<t_{b-c_{t}+1}$, or $c_{t}=0$ if $t>t_{b}$. Then, for all $z \in\left\{c_{t}, c_{t}+1, \ldots, b\right\}$, we have $t_{b-z}<t$, and thus $t+b-z<L t$; and for all $z^{\prime}<c_{t}$, we have $t_{b-z^{\prime}}>t$, and thus $t+b-z^{\prime}>L t$. Using the results from (11), (12) and (16), 
we have that for all $n \geq N_{F}$,

$$
\begin{aligned}
& \mathbb{P}\left(\mathcal{E}_{t}^{\beta}\right) \\
& =\mathbb{P}\left(\frac{A(-t-b,-b-1)}{n_{0}}-X_{F}(-t-b,-1)>0\right) \\
& =\sum_{a=0}^{t+b-c_{t}} \mathbb{P}\left(\sum_{\tau=-t-b}^{-1} X_{F}(\tau)=a\right) \mathbb{P}\left(A(-t-b,-b-1)>a n_{0}\right) \\
& \leq(t+b+1) \max _{0 \leq a \leq t+b-c_{t}}\left\{\mathbb{P}\left(\sum_{\tau=-t-b}^{-1} X_{F}(\tau)=a\right)\right. \\
& \left.\times \mathbb{P}\left(A(-t-b,-b-1)>a n_{0}\right)\right\} \\
& \leq(t+b+1) \max \left\{\max _{a \in\{0,1, \ldots, t-1\}}\left\{\mathbb{P}\left(\sum_{\tau=-t-b}^{-1} X_{F}(\tau)=a\right)\right\},\right. \\
& \max _{a \in\left\{0, \ldots, b-c_{t}\right\}}\left\{\mathbb{P}\left(\sum_{\tau=-t-b}^{-1} X_{F}(\tau)=t+a\right)\right. \\
& \left.\left.\times \mathbb{P}\left(A(-t-b,-b-1)>(t+a) n_{0}\right)\right\}\right\} \\
& \stackrel{(a)}{\leq}(t+b+1) \max \left\{2^{t+b}\left(\frac{n}{1-q}\right)^{7 b H} e^{-n(b+1) I_{X}},\right. \\
& \max _{a \in\left\{0, \ldots, b-c_{t}\right\}}\left\{\mathbb{P}\left(\sum_{\tau=-t-b}^{-1} X_{F}(\tau)=t+a\right)\right. \\
& \left.\left.\times \mathbb{P}\left(A(-t-b,-b-1)>(t+a) n_{0}\right)\right\}\right\} \\
& \stackrel{(b)}{\leq}(t+b+1) 2^{t+b}\left(\frac{n}{1-q}\right)^{7 b H} e^{(H(t+b)-1) \theta^{*}} \\
& \times \max \left\{e^{-n(b+1) I_{X}}, \max _{a \in\left\{0, \ldots, b-c_{t}\right\}}\left\{e^{-n\left(I_{A}(t, a)+(b-a) I_{X}\right)}\right\}\right\} \\
& \stackrel{(c)}{\leq} C_{3} n^{7 b H} e^{-n \min \left\{(b+1) I_{X}, \min _{z \in\left\{c_{t}, c_{t}+1, \ldots, b\right\}}\left\{I_{A}(t, b-z)+z I_{X}\right\}\right\}},
\end{aligned}
$$

where $C_{3} \triangleq\left(t^{*}+b+1\right) 2^{t^{*}+b}\left(\frac{1}{1-q}\right)^{7 b H} e^{\left(H\left(t^{*}+b\right)-1\right) \theta^{*}}$, (a) is from the monotonicity of the right hand side of (12), (b) is from (12) and (16), and (c) is from changing variable by setting $z=b-a$.

Recall that

$$
\begin{aligned}
I_{0}(b) \triangleq \min \{( & (b+1) I_{X}, \\
& \min _{0 \leq c \leq b}\left\{\inf _{t>t_{b-c}} I_{A}(t, b-c)+c I_{X}\right\}, \\
& \left.\min _{c \in \Psi_{b}}\left\{I_{A}\left(t_{b-c}, b-c\right)+(c+1) I_{X}\right\}\right\} .
\end{aligned}
$$


Hence, for all $n \geq N_{4} \triangleq \max \left\{N_{3}, N_{F}\right\}$, we have

$$
\begin{aligned}
\mathbb{P}\left(\mathcal{E}_{t}^{F} \cap \mathcal{E}_{t}^{P M}\right) & \leq \mathbb{P}\left(\mathcal{E}_{t}^{F}\right) \\
& \stackrel{(a)}{\leq} 1-\mathbb{P}\left(\mathcal{E}_{t}^{\alpha}\right)+\mathbb{P}\left(\mathcal{E}_{t}^{\beta}\right) \\
& \stackrel{(b)}{\leq} C_{4} n^{7 b H} e^{-n I_{0}(b)}
\end{aligned}
$$

where $C_{4} \triangleq \max \left\{C_{2} t^{*}, C_{3}\right\}$, (a) is from (14), and (b) is from (15) and the above result.

Next, we consider any $t_{b-c} \in\left\{t_{b-c^{\prime}} \mid c^{\prime} \in \Psi_{b}\right\}$. For these values of $t$, we will use the dominance property over both the FBS policy and the perfect-matching policy, i.e., $\mathcal{E}_{t} \subseteq \mathcal{E}_{t}^{F}$ and $\mathcal{E}_{t} \subseteq \mathcal{E}_{t}^{P M}$. Recall that we have $t_{b-c}=\frac{b-c}{L-1}>0$, and thus

$$
t_{b-c}+b-c=L t_{b-c} .
$$

We first split $\mathbb{P}\left(\mathcal{E}_{t_{b-c}}^{F} \cap \mathcal{E}_{t_{b-c}}^{P M}\right)$ as

$$
\mathbb{P}\left(\mathcal{E}_{t_{b-c}}^{F} \cap \mathcal{E}_{t_{b-c}}^{P M}\right) \leq K_{1}+K_{2}
$$

where

$$
\begin{aligned}
K_{1} \triangleq & \mathbb{P}\left(\mathcal{E}_{t_{b-c}}^{F} \cap \mathcal{E}_{t_{b-c}}^{P M},\right. \\
& \left.A\left(-t_{b-c}-b,-b-1\right)<\left(t_{b-c}+b-c\right) n_{0}\right) \\
\leq & \mathbb{P}\left(\mathcal{E}_{t_{b-c}}^{F}, A\left(-t_{b-c}-b,-b-1\right)<\left(t_{b-c}+b-c\right) n_{0}\right) \\
\leq & 1-\mathbb{P}\left(\mathcal{E}_{t_{b-c}}^{\alpha}\right)+K_{1}^{\prime}, \\
K_{1}^{\prime} \triangleq & \mathbb{P}\left(\mathcal{E}_{t_{b-c}}^{\beta}, A\left(-t_{b-c}-b,-b-1\right)<\left(t_{b-c}+b-c\right) n_{0}\right),
\end{aligned}
$$

and

$$
\begin{aligned}
K_{2} \triangleq & \mathbb{P}\left(\mathcal{E}_{t_{b-c}}^{F} \cap \mathcal{E}_{t_{b-c}}^{P M},\right. \\
& \left.A\left(-t_{b-c}-b,-b-1\right) \geq\left(t_{b-c}+b-c\right) n_{0}\right) \\
\leq & \mathbb{P}\left(\mathcal{E}_{t_{b-c}}^{P M}, A\left(-t_{b-c}-b,-b-1\right) \geq\left(t_{b-c}+b-c\right) n_{0}\right) .
\end{aligned}
$$

In the above derivation, we use the dominance over the FBS policy and the perfect-matching policy for $K_{1}$ and $K_{2}$, respectively. 
Similarly, using the results from (11), (12), and (16), we have that for all $n \geq N_{F}$,

$$
\begin{aligned}
K_{1}^{\prime}= & \mathbb{P}\left(\frac{A\left(-t_{b-c}-b,-b-1\right)}{n_{0}}-\sum_{\tau=-t_{b-c}-b}^{-1} X_{F}(\tau)>0,\right. \\
& \left.A\left(-t_{b-c}-b,-b-1\right)<\left(t_{b-c}+b-c\right) n_{0}\right) \\
& \sum_{a=0}^{t_{b-c}+b-c-1}\left(\mathbb{P}\left(\sum_{\tau=-t_{b-c}-b}^{-1} X_{F}(\tau)=a\right)\right. \\
\leq\left(t_{b-c}+b-c\right) & \left.\quad \times \mathbb{P}\left(A\left(-t_{b-c}-b,-b-1\right)>a n_{0}\right)\right) \\
& \left.\times \mathbb{P}\left(A\left(-t_{b-c}-b,-b-1\right)>a t_{0}\right)\right\} \\
\leq & C_{3} n^{7 b H} e^{-n \min \left\{(b+1) I_{X}, \min _{z \in\{c+1, \ldots, b\}}\left\{I_{A}\left(t_{b-c}, b-z\right)+z I_{X}\right\}\right\}},
\end{aligned}
$$

and using the results from (13) and (16), we have that for all $n \geq N_{P M}$,

$$
\begin{aligned}
& K_{2} \leq \mathbb{P}\left(\mathcal{E}_{t_{b-c}}^{P M} \mid A\left(-t_{b-c}-b,-b-1\right) \geq\left(t_{b-c}+b-c\right) n_{0}\right) \\
& \times \mathbb{P}\left(A\left(-t_{b-c}-b,-b-1\right) \geq\left(t_{b-c}+b-c\right) n_{0}\right) \\
& \stackrel{(a)}{\leq} \mathbb{P}\left(\mathcal{E}_{t_{b-c}}^{P M} \mid A\left(-t_{b-c}-b,-b-1\right)=\text { Lnt }_{b-c}\right) \\
& \times e^{-n I_{A}\left(t_{b-c}, b-c\right)} e^{\left(H\left(t_{b-c}+b-c\right)-1\right) \theta^{*}} \\
& \stackrel{(b)}{\leq} \mathbb{P}\left(\sum_{\tau=-t_{b-c}-b}^{-1} X_{P M}(\tau)<t_{b-c}+b-c\right) \\
& \times e^{-n I_{A}\left(t_{b-c}, b-c\right)} e^{\left(H\left(t_{b-c}+b-c\right)-1\right) \theta^{*}} \\
& \leq \sum_{a=0}^{t_{b-c}+b-c-1} \mathbb{P}\left(\sum_{\tau=-t_{b-c}-b}^{-1} X_{P M}(\tau)=a\right) \\
& \times e^{-n I_{A}\left(t_{b-c}, b-c\right)} e^{\left(H\left(t_{b-c}+b-c\right)-1\right) \theta^{*}} \\
& \leq\left(t_{b-c}+b-c\right) \max _{a \in\left\{0, \ldots, t_{b-c}+b-c-1\right\}} \mathbb{P}\left(\sum_{\tau=-t_{b-c}-b}^{-1} X_{P M}(\tau)=a\right) \\
& \times e^{-n I_{A}\left(t_{b-c}, b-c\right)} e^{\left(H\left(t_{b-c}+b-c\right)-1\right) \theta^{*}} \\
& \stackrel{(c)}{\leq}\left(t_{b-c}+b-c\right) 2^{t+3 b} e^{\left(H\left(t_{b-c}+b-c\right)-1\right) \theta^{*}} n^{b} \\
& \times e^{-n\left(I_{A}\left(t_{b-c}, b-c\right)+(c+1) I_{X}\right)} \\
& \leq C_{5} n^{b} e^{-n\left(I_{A}\left(t_{b-c}, b-c\right)+(c+1) I_{X}\right)},
\end{aligned}
$$


where $C_{5} \triangleq\left(t^{*}+b\right) 2^{t^{*}+3 b} e^{\left(H\left(t^{*}+b\right)-1\right) \theta^{*}}$, (a) is from [16), (b) is because $t_{b-c}=\frac{b-c}{L-1}$ and the perfectmatching policy serves exactly $n$ packets in each time-slot when $A\left(-t_{b-c}-b,-b-1\right)=L n t_{b-c}$, and (c) is from (13) and the monotonicity of its right hand side.

Hence, from the above results, (15), and (18), we have that for any $t_{b-c}$ with $c \in \Psi_{b}$, and for all $n \geq N_{5} \triangleq \max \left\{N_{3}, N_{F}, N_{P M}\right\}$,

$$
\mathbb{P}\left(\mathcal{E}_{t_{b-c}}^{F} \cap \mathcal{E}_{t_{b-c}}^{P M}\right) \leq C_{6} n^{7 b H} e^{-n I_{0}(b)},
$$

where $C_{6} \triangleq \max \left\{C_{2} t^{*}, C_{3}, C_{5}\right\}$.

Summing over $t=1$ to $t^{*}$, we have

$$
\begin{aligned}
P_{1} & =\sum_{t=1}^{t^{*}} \mathbb{P}\left(L(-b)=-t_{b-c}-b-1, \mathcal{E}_{t}\right) \\
& \leq C_{1} n^{7 b H} e^{-n I_{0}(b)}
\end{aligned}
$$

for all $n \geq N_{1} \triangleq \max \left\{N_{4}, N_{5}\right\}$, where $C_{1} \triangleq C_{6} t^{*}$.

Part 2: We want to show that there exists an $N_{2}>0$ such that for all $n \geq N_{2}$, we have

$$
P_{2} \leq 4 e^{-n I_{0}(b)}
$$

Before we proceed, we first provide an informal discussion on the intuition behind. In Part 1, as we have seen, the delay-violation event could occur due to both bursty arrivals and sluggish services. However, when time interval $t$ is large enough, from Assumption 3 , we know that the total arrivals to the system will not deviate far away from its mean $n p t$ during an interval of $t$ time-slots for large $n$. On the other hand, if FBS can find a schedule to serve the HOL frame in each time-slot during an interval of $t$ time-slots, the total service can sum up to $(n-L h) t$. Hence, the delay-violation event occurs mostly due to sluggish services when $t$ is large.

Let $R_{0}$ be the empty space in the end-of-line frame at the end of time-slot $t_{1}$. Then, let $A_{F}^{R_{0}}\left(t_{1}, t_{2}\right)$ denote the number of new frames created from time-slot $t_{1}$ to $t_{2}$, including any partially-filled frame in time-slot $t_{2}$, but excluding the partially-filled frame in time-slot $t_{1}$. Also, let $A_{F}\left(t_{1}, t_{2}\right)=A_{F}^{R_{0}}\left(t_{1}, t_{2}\right)$, if $R_{0}=0$. As in the proof for Theorem 2 of [10], for any fixed real number $\hat{p} \in(p, 1)$, we consider the arrival process $\hat{A}(\cdot)$, by adding extra dummy arrivals to the original arrival process $A(\cdot)$. The resulting arrival process $\hat{A}(\cdot)$ is simple, and has the following property:

$$
\hat{A}(\tau)= \begin{cases}\hat{p} n, & \text { if } A(\tau) \leq \hat{p} n \\ L n, & \text { if } A(\tau)>\hat{p} n\end{cases}
$$


Hence, if we can find an upper bound on $\hat{A}_{F}(-t-b,-b-1)$, by our construction, then it is also an upper bound on $A_{F}(-t-b,-b-1)$.

Consider any $t \geq t^{*}$. Let $B=\left\{b_{1}, b_{2}, \ldots, b_{|B|}\right\}$ be the set of time-slots in the interval from $-t-b$ to $-b-1$ when $\hat{A}(\tau)=L n$. Given $L(-b)=-t-b-1$, from Corollary 2 of [11], we have that,

$$
\begin{aligned}
\hat{A}_{F}(- & t-b,-b-1) \\
\leq & \sum_{r=1}^{|B|-1}\left\lceil\frac{\hat{A}\left(b_{r}+1, b_{r+1}-1\right)}{n_{0}}\right\rceil+\sum_{r=1}^{|B|}\left\lceil\frac{\hat{A}\left(b_{r}, b_{r}\right)}{n_{0}}\right] \\
& +\left\lceil\frac{\hat{A}\left(-t-b, b_{1}-1\right)}{n_{0}}\right]+\left\lceil\frac{\hat{A}\left(b_{|B|}+1,-b-1\right)}{n_{0}}\right] \\
\leq & \sum_{r=1}^{|B|-1} \frac{\hat{A}\left(b_{r+1}-1-b_{r}\right) \hat{p} n}{n_{0}}+|B|-1+\sum_{r=1}^{|B|} \frac{L n}{n_{0}}+|B| \\
& \quad+\frac{\left(b_{1}+t+b\right) \hat{p} n}{n_{0}}+1+\frac{\left(-b-1-b_{|B|}\right) \hat{p} n}{n_{0}}+1 \\
\leq & \frac{(t-|B|) \hat{p} n}{n_{0}}+|B| \frac{L n}{n_{0}}+2|B|+1 \\
\leq & \frac{n}{n_{0}}(\hat{p} t+(L+2)|B|+1) .
\end{aligned}
$$

From Assumption 3 on the arrival process we know that for large enough $n$ and $t,|B|$ can be made less than an arbitrarily small fraction of $t$. Further, we can show that for $n \geq \frac{(2+\hat{p}) H}{1-\hat{p}}, t>\frac{6}{1-\hat{p}}$ and $|B|<\frac{1-\hat{p}}{6(L+2)} t$, we have $\hat{A}_{F}(-t-b,-b-1)<\left(\frac{2+\hat{p}}{3}\right) t$. This is derived by substituting the values of $n, t$ and $|B|$ in the equation above,

$$
\begin{aligned}
A_{F}( & -t-b,-b-1) \\
& \leq \hat{A}_{F}(-t-b,-b-1) \\
& \leq \frac{n}{n_{0}}(\hat{p} t+(L+2)|B|+1) \\
& <\frac{2+\hat{p}}{1+2 \hat{p}}\left(\hat{p} t+\frac{1-\hat{p}}{3} t\right) \\
& \leq\left(\frac{2+\hat{p}}{3}\right) t .
\end{aligned}
$$


Then, it follows that

$$
\begin{aligned}
& \mathbb{P}\left(A_{F}(-t-b,-b-1) \geq\left(\frac{2+\hat{p}}{3}\right) t\right. \\
& L(-b)=-t-b-1) \\
& =1-\mathbb{P}\left(A_{F}(-t-b,-b-1)<\left(\frac{2+\hat{p}}{3}\right) t\right. \\
& \quad L(-b)=-t-b-1) \\
& \leq 1-\mathbb{P}\left(|B| \leq \frac{1-\hat{p}}{6(L+2)} t\right) \\
& \leq e^{-n t I_{B}\left(\hat{p}-p, \frac{1-\hat{p}}{6(L+2)}\right)}
\end{aligned}
$$

for all $n \geq N_{6} \triangleq \max \left\{N_{B}\left(\hat{p}-p, \frac{1-\hat{p}}{6(L+2)}\right), \frac{(2+\hat{p}) H}{1-\hat{p}}\right\}$ and $t \geq T_{1}$, where the last inequality is from Assumption 3 and (9).

Next, we state a lemma that will be used in the rest of the proof.

Lemma 12: Let $X_{i}$ be a sequence of binary random variables satisfying

$$
\mathbb{P}\left(X_{i}=0\right)<c(n) e^{-n d}, \text { for all } i,
$$

where $c(n)$ is a polynomial in $n$ of finite degree. Let $N_{X}$ be such that $c(n)<e^{\frac{n d}{2}}$ for all $n \geq N_{X}$. Then, for any real number $a \in(0,1)$, we have

$$
\mathbb{P}\left(\sum_{i=1}^{t} X_{i}<(1-a) t\right) \leq e^{-\frac{t n a d}{3}}
$$

for all $n \geq \max \left\{\frac{12}{a d}, N_{X}\right\}$

The proof follows immediately from Lemma 1 of [10].

We know from Lemma 6 of [10] that for each time-slot $\tau, X_{F}(\tau)=0$ with probability less than $\left(\frac{n}{1-q}\right)^{7 H} e^{-n I_{X}}$ for all $n \geq N_{F}$. Hence, from Lemma 12, we have that there exists an $N_{7}>N_{F}$ such that,

$$
\begin{aligned}
& \mathbb{P}\left(X_{F}(-t-b,-1)<\left(\frac{2+\hat{p}}{3}\right) t, L(-b)=-t-b-1\right) \\
& \leq \mathbb{P}\left(X_{F}(-t-b,-1)<\left(\frac{2+\hat{p}}{3}\right)(t+b),\right. \\
& \quad L(-b)=-t-b-1) \\
& \leq e^{-n(t+b)\left(\frac{1-\hat{p}}{9}\right) I_{X}} \\
& \leq e^{-n t \frac{(1-\hat{p}) I_{X}}{9}},
\end{aligned}
$$

for all $n \geq N_{7}$ and $t>0$. 
From (19) and (20), we have that for all $n \geq N_{8} \triangleq \max \left\{N_{6}, N_{7}\right\}$ and $t \geq T_{1}$,

$$
\begin{aligned}
\mathbb{P}( & A_{F}(-t-b,-b-1)-X_{F}(-t-b,-1)>0, \\
& L(-b)=-t-b-1) \\
\leq & 1-\left(1-e^{-n t\left(\frac{1-\hat{p}}{9}\right) I_{X}}\right)\left(1-e^{-n t I_{B}\left(\hat{p}-p, \frac{1-\hat{p}}{6(L+2)}\right)}\right) \\
\leq & 2 e^{-n t I_{B X}},
\end{aligned}
$$

where the last inequality is from (10).

Then, summing over all $t \geq t^{*}$, we have that for all $n \geq N_{2} \triangleq \max \left\{N_{8},\left\lceil\frac{\log 2}{I_{B X}}\right\rceil\right\}$,

$$
\begin{aligned}
P_{2} & \leq \sum_{t=t^{*}}^{\infty} \mathbb{P}\left(\mathcal{E}_{t}^{F}\right) \\
& \leq \sum_{t=t^{*}}^{\infty} \mathbb{P}(L(-b)=-t-b-1, \\
& \left.\quad A_{F}(-t-b,-b-1)>X_{F}(-t-b,-1)\right) \\
& \leq \sum_{t=t^{*}}^{\infty} 2 e^{-n t I_{B X}} \\
& \leq \frac{2 e^{-n t^{*} I_{B X}}}{1-e^{-n I_{B X}}} \\
& \stackrel{(a)}{\leq} 4 e^{-n t^{*} I_{B X}} \\
& \stackrel{(b)}{\leq} 4 e^{-n I_{0}(b)},
\end{aligned}
$$

where (a) is from our choice of $N_{2}$, and (b) is from (8).

Combining both parts, we complete the proof for the case of $L>1$.

Now, we consider the case of $L=1$. We want to show that for any fixed integer $b \geq 0$, the rate-function attained by policy $\mathbf{P}$ is no smaller than $(b+1) I_{X}$.

Similarly, we fix a finite time $t^{\prime}$ as

$$
t^{\prime} \triangleq \max \left\{T_{1},\left\lceil\frac{(b+1) I_{X}}{I_{B X}}\right\rceil\right\}
$$

Using the dominance property over the FBS policy and the perfect-matching policy, we split the summation in (6) as

$$
\mathbb{P}(W(0)>b) \leq P_{1}^{\prime}+P_{2}^{\prime},
$$

where $P_{1}^{\prime} \triangleq \sum_{t=1}^{t^{\prime}} \mathbb{P}\left(\mathcal{E}_{t}^{F} \cap \mathcal{E}_{t}^{P M}\right)$, and $P_{2}^{\prime} \triangleq \sum_{t=t^{\prime}}^{\infty} \mathbb{P}\left(\mathcal{E}_{t}^{F} \cap \mathcal{E}_{t}^{P M}\right)$. 
We divide the proof into two parts. In Part 1, we show that there exists a finite $N_{1}^{\prime}>0$ such that for all $n \geq N_{1}^{\prime}$, we have

$$
P_{1}^{\prime} \leq C_{1}^{\prime} n^{b} e^{-n(b+1) I_{X}} .
$$

Then, in Part 2, we show that there exists a finite $N_{2}^{\prime}>0$ such that for all $n \geq N_{2}^{\prime}$, we have

$$
P_{2}^{\prime} \leq 4 e^{-n(b+1) I_{X}}
$$

Finally, combining both parts, we have

$$
\mathbb{P}(W(0)>b) \leq\left(C_{1}^{\prime} n^{b}+4\right) e^{-n(b+1) I_{X}},
$$

for all $n \geq N^{\prime} \triangleq \max \left\{N_{1}^{\prime}, N_{2}^{\prime}\right\}$. By taking logarithm and limit as $n$ goes to infinity, we obtain $\liminf _{n \rightarrow \infty} \frac{-1}{n} \log \mathbb{P}(W(0)>b) \geq(b+1) I_{X}$, and thus the desired results.

For Part 2, by applying the same argument as in the case of $L>1$, we can show that there exists a finite $N_{2}^{\prime}>0$ such that for all $n \geq N_{2}^{\prime}$, we have $P_{2}^{\prime} \leq 4 e^{-n(b+1) I_{X}}$. Hence, it remains to show $P_{1}^{\prime} \leq C_{1}^{\prime} n^{b} e^{-n(b+1) I_{X}}$.

Consider any $t \in\left\{1,2, \ldots, t^{\prime}\right\}$. Note that the total number of packet arrivals to the system during the interval of $[-t-b,-b-1]$ can never exceed $n t$ when $L=1$, then event $\mathcal{E}_{t}^{P M}$ does not occur if the total number of time-slots when a perfect-matching can be found during the interval of $[-t-b,-1]$ is no smaller than $t$, i.e., $\sum_{\tau=-t-b}^{-1} X_{P M}(\tau) \geq t$. Thus, we have $\mathcal{E}_{t}^{P M} \subseteq\left\{L(b)=-t-b-1, \sum_{\tau=-t-b}^{-1} X_{P M}(\tau)<t\right\}$. Then, we have

$$
\begin{aligned}
& \mathbb{P}\left(\mathcal{E}_{t}^{F} \cap \mathcal{E}_{t}^{P M}\right) \\
& \leq \mathbb{P}\left(\mathcal{E}_{t}^{P M}\right) \\
& \leq \mathbb{P}\left(\sum_{\tau=-t-b}^{-1} X_{P M}(\tau)<t\right) \\
& \leq t \max _{a \in\{0, \ldots, t-1\}} \mathbb{P}\left(\sum_{\tau=-t-b}^{-1} X_{P M}(\tau)=a\right) \\
& \stackrel{(a)}{\leq} t^{\prime} 2^{t^{\prime}+3 b} n^{b} e^{-n(b+1) I_{X}} \\
& \leq C_{3}^{\prime} n^{b} e^{-n(b+1) I_{X}},
\end{aligned}
$$

where $C_{3}^{\prime} \triangleq t^{\prime} 2^{t^{\prime}+3 b}$, and (a) is from (13) and the monotonicity of its right hand side.

Let $C_{1}^{\prime} \triangleq t^{\prime} C_{3}^{\prime}$. Summing over all $t \in\left\{1, \ldots, t^{\prime}\right\}$, we have

$$
P_{1}^{\prime} \leq C_{1}^{\prime} n^{b} e^{-n(b+1) I_{X}} .
$$


Combining both parts, we complete the proof for the case of $L=1$. Then, combining both cases of $L>1$ and $L=1$, the result of the theorem follows.

\section{APPENDIX D}

\section{PROOF OF LEMMA 3}

Suppose policy $\mathbf{P}$ satisfies the sufficient condition in Theorem 2, We first want to show that policy $\mathbf{P}$ dominates the version of the FBS policy described in Section IV-A The proof follows a similar argument as in the proof of Lemma 7 in [10].

Consider two queueing systems, $\bar{Q}_{1}$ and $\bar{Q}_{2}$, both of which have the same arrival and channel realizations. We assume that $\bar{Q}_{1}$ adopts policy $\mathbf{P}$ and $\bar{Q}_{2}$ adopts the FBS policy. Recall that the weight of a packet $p$ in time-slot $t$ is defined as $\hat{w}(p)=t-t_{p}+\frac{L+1-x_{p}}{(L+1)}+\frac{n+1-q(p)}{(L+1)(n+1)}$. For two packets $p_{1}$ and $p_{2}$, we say $p_{1}$ is older than $p_{2}$ if $\hat{w}\left(p_{1}\right)>\hat{w}\left(p_{2}\right)$.

Let $R_{i}(t)$ represent the set of packets present in the system $\bar{Q}_{i}$ at the end of time-slot $t$, for $i=1,2$. Then, it suffices to show that $R_{1}(t) \subseteq R_{2}(t)$ for all time $t$. We let $A(t)$ denote the set of packets that arrive at time $t$. Let $X_{i}(t)$ denote the set of packets that depart the system $\bar{Q}_{i}$ at time t, for $i=1,2$. Hence, we have $R_{i}(t+1)=\left(R_{i}(t) \cup A(t+1)\right) \backslash X_{i}(t+1)$, for $i=1,2$.

We then proceed the proof by contradiction. Suppose that $R_{1}(t) \nsubseteq R_{2}(t)$ for some time $t$. Without loss of generality, we assume that $\tau$ is the first time such that $R_{1}(\tau) \nsubseteq R_{2}(\tau)$ occurs. Hence, there must exist a packet, say $p$, such that $p \in R_{1}(\tau)$ and $p \notin R_{2}(\tau)$. Because $\tau$ is the first time when such an event occurs, packet $p$ must depart from the system $\bar{Q}_{2}$ in time-slot $\tau$, i.e., $p \in X_{2}(\tau)$.

Let $B_{i}(v)$ denote the set of packets in $R_{i}(\tau-1) \cup A(\tau)$ with weight greater than or equal to $v$, for $i=1,2$. Clearly, we have $B_{1}(v) \subseteq B_{2}(v)$ for all $v$, as $R_{1}(\tau-1) \subseteq R_{2}(\tau-1)$ by assumption. Since packet $p$ is served in the system $\bar{Q}_{2}$ in time-slot $\tau$, we know from the operations of FBS that all packets in $B_{2}(\hat{w}(p))$ must also be served in time-slot $\tau$. This is because packet $p$ is part of the HOL frame in time-slot $\tau$ (as packet $p$ is served in time-slot $\tau$ ), and all packets with a weight greater than $\hat{w}(p)$ must be filled to the frames with higher priority than packet $p$ and thus should also belong to the HOL frame in time-slot $\tau$. This further implies that in the system $\bar{Q}_{1}$, there exists a feasible schedule that can match all packets in $B_{1}(\hat{w}(p))$, since $B_{1}(\hat{w}(p)) \subseteq B_{2}(\hat{w}(p))$ and both systems have the same channel realizations.

Now, from the sufficient condition in Theorem 2, policy $\mathbf{P}$ will serve all packets in $B_{1}(\hat{w}(p))$, including packet $p$. This contradicts with the hypothesis that packet $p$ is not served (by policy $\mathbf{P}$ ) in the system $\bar{Q}_{1}$ in time-slot $\tau$ (i.e., $p \notin R_{1}(\tau)$ ). 
So far, we have shown that for any given sample path and for any value of $h$, by the end of any time-slot $t$, policy $\mathbf{P}$ has served every packet that the FBS policy has served.

Next, we want to show that policy $\mathbf{P}$ dominates the version of the perfect-matching policy described in Section IV-A Note that in each time-slot, the packets served by the perfect-matching policy are the oldest packets in the system. The difference between FBS and the perfect-matching is the following. The HOL frame that can be served by FBS has at most $L h$ packets from each queue and has at most $n_{0}=n-L h$ packets from the system, while the set of packets that can be served by the perfect-matching policy has at most one packet from each queue and has at most $n$ packets from the system. Following a similar argument as above for the FBS policy, we can show that for any given sample path, by the end of any time-slot $t$, policy $\mathbf{P}$ has served every packet that the perfect-matching policy has served. This completes the proof.

\section{APPENDIX E}

\section{Proof of Proposition 4}

We first prove that DWM- $n$ policy is an OPF policy and is thus rate-function delay-optimal. The proof follows immediately from a property of the MVM in bipartite graphs. We restate this property in the following lemma.

Lemma 13 (Lemma 6 of [15]): Consider a bipartite graph, and the $k$ heaviest vertices, for some $k$. If there is a matching that matches all the heaviest $k$ vertices, then any MVM matches all of them too.

Since DWM- $n$ policy finds an MVM in the constructed bipartite graph, Lemma 13 implies that for any $k \in\{1,2, \ldots, n\}$, if the $k$ oldest packets can be served by some scheduling policy, then DWM- $n$ policy can serve these $k$ packets as well. This completes the first part of the proof.

Next, we prove that DWM- $n$ policy has a complexity of $O\left(n^{2.5} \log n\right)$. Note that in order to select the $n$ oldest packets in the system, it is sufficient to sort the $n^{2}$ packets picked by DWM policy, i.e., the $n$ oldest packets of each of the $n$ queues, as no other packets can be among the $n$ oldest packets in the system. The complexity of sorting $n^{2}$ packets [23] is $O\left(n^{2} \log n\right)$. Given the $n$ oldest packets in the system, DWM- $n$ policy constructs an $n \times n$ bipartite graph and finds an MVM [15] in $O\left(n^{2.5} \log n\right)$ time. Hence, the overall complexity of DWM- $n$ is $O\left(n^{2.5} \log n\right)$, which completes the proof.

\section{APPENDIX F}

\section{Proof OF Proposition 5}

The following simple counter-example shows that DWM- $n$ cannot stabilize a feasible arrival rate vector, and is thus not throughput-optimal in general. 
Consider a system with two queues and two servers, i.e., a system with $n=2$. We assume the i.i.d. ON-OFF channel model as in Assumption 4 i.e., each server is connected to each queue with probability $q \in(0,1)$, and is disconnected otherwise. In each time-slot, a server can serve at most one packet of a queue that is connected to this server. In such a system, the optimal throughput region can be described as $\Lambda^{*}=\left\{\lambda \mid \lambda_{1} \leq 2 q, \lambda_{2} \leq 2 q\right.$, and $\left.\lambda_{1}+\lambda_{2} \leq 2\left(2 q-q^{2}\right)\right\}$, where the first two inequalities are obvious, and the last inequality is due to the following. For each of the two servers, the probability that at least one queue is connected to the server is $2 q-q^{2}$, hence, the service each server can provide is $2 q-q^{2}$, and the total (effective) capacity is thus $2\left(2 q-q^{2}\right)$. Note that any arrival rate vector $\lambda$ strictly inside the optimal throughput region $\Lambda^{*}$, is feasible.

Next, we construct an arrival process as follows. Consider a frame consisting of two time-slots. In each frame, there are packet arrivals to the system with probability $p \in(0,1)$, and no arrivals otherwise. In a frame that has arrivals, there are $K$ packet arrivals to queue $Q_{1}$ and no arrivals to queue $Q_{2}$ in the first time-slot, and there are no arrivals to queue $Q_{1}$ and $K$ packet arrivals to queue $Q_{2}$ in the second time-slot, where we assume that $K \geq 4$. This type of arrival process yields an arrival rate vector of $\lambda^{*}=\left[\frac{p K}{2}, \frac{p K}{2}\right]$. It is easy to check that $\lambda^{*}$ is feasible, if $p K \leq 4 q-2 q^{2}$.

Now, we characterize an upper bound of the service rate under DWM- $n$ policy. Recall that DWM- $n$ considers only the $n$ oldest packets in the system and maximizes the sum of the delays of the packets scheduled over these $n$ packets, and no other packets will be scheduled. Hence, in each time-slot, DWM$n$ considers only the two oldest packets in the system. Consider any time-slot $t_{1}$, where $K-1$ out of the $K$ packets arriving to queue $Q_{1}$ in the same time-slot are still waiting in the system. The other one packet could have been scheduled with a packet in $Q_{2}$, or with a packet that arrived to $Q_{1}$ earlier, or it could have been scheduled alone in a time-slot before $t_{1}$. Note that the first $K-2$ packets out of these $K-1$ packets cannot be scheduled with packets in queue $Q_{2}$, due to the operations of DWM- $n$. Hence, in any time-slot $t_{2}$ before these $K-1$ packets are completely evacuated, each server must serve queue $Q_{1}$ if this server is connected to queue $Q_{1}$, and no server will serve $Q_{2}$ even if this server is connected to queue $Q_{2}$, as the packets of $Q_{2}$ are not among the two oldest packets in the system in such time-slot $t_{2}$. Hence, the expected service rate for these $K-2$ packets is $2 q$, and it thus takes $\frac{K-2}{2 q}$ time-slots on average to evacuate the $K-2$ packets. Similarly, it takes $\frac{K-2}{2 q}$ time-slots on average to evacuate such $K-2$ packets in queue $Q_{2}$. Therefore, the total service rate of the system under DWM- $n$ is no greater than $\frac{2 K}{\frac{2(K-2)}{2 q}}=\frac{2 q K}{K-2}$. It is clear that the system is unstable if the total arrival rate is greater than the total service rate, i.e., $p K>\frac{2 q K}{K-2}$. Then, by choosing $p=\frac{17}{96}, q=\frac{1}{2}$ and $K=8$, we obtain a feasible arrival rate vector $\lambda^{*}$ that cannot be stabilized by DWM- $n$. This completes the proof. 


\section{APPENDIX G}

\section{PROOF OF THEOREM 6}

Suppose that the sufficient condition is satisfied under policy $\mathbf{P}$, i.e., there exists a constant $M>0$ such that in any time-slot $t$ and for all $j \in\{1,2, \ldots, n\}$, queue $Q_{i(j, t)}$ satisfies that $W_{i(j, t)}(t) \geq Z_{r, M}(t)$ for all $r \in \Gamma_{j}(t)$ such that $Q_{r}(t) \geq M$. We want to show that policy $\mathbf{P}$ can stabilize any arrival rate vector $\lambda$ strictly inside the optimal throughput region $\Lambda^{*}$.

Recall that $Q_{i}(t)$ denotes the queue length of $Q_{i}$ at the beginning of time-slot $t, Z_{i, l}(t)$ denotes the delay of the $l$-th packet of $Q_{i}$ at the beginning of time-slot $t, W_{i}(t)$ denotes the HOL delay of $Q_{i}$ at the beginning of time-slot $t$, and $C_{i, j}(t)$ denotes the connectivity between queue $Q_{i}$ and server $S_{j}$ in time-slot $t$. Let $Y_{i, j}(t)$ denote the service of queue $Q_{i}$ received from server $S_{j}$ in time-slot $t$, i.e., $Y_{i, j}(t)=C_{i, j}(t)$ if server $S_{j}$ is allocated to serve queue $Q_{i}$, and $Y_{i, j}(t)=0$ otherwise. We define the random process describing the behavior of the underlying system as $\mathcal{X}=(\mathcal{X}(t), t=0,1,2, \ldots)$, where

$$
\begin{gathered}
\mathcal{X}(t) \triangleq\left\{\left(Z_{i, 1}(t), Z_{i, 2}(t), \ldots, Z_{i, Q_{i}(t)}(t)\right), 1 \leq i \leq n ;\right. \\
\left.C_{i, j}(t), 1 \leq i \leq n, 1 \leq j \leq n\right\} .
\end{gathered}
$$

The norm of $\mathcal{X}(t)$ is defined as $\|\mathcal{X}(t)\| \triangleq \sum_{1 \leq i \leq n} Q_{i}(t)+\sum_{1 \leq i \leq n} W_{i}(t)$. Let $\mathcal{X}^{(x)}$ denote a process $\mathcal{X}$ with an initial condition such that

$$
\left\|\mathcal{X}^{(x)}(0)\right\|=x
$$

The following Lemma was derived in [24] for continuous-time countable Markov chains, and it follows from more general results in [25] for discrete-time countable Markov chains.

Lemma 14: Suppose that there exist a real number $\epsilon>0$ and an integer $T>0$ such that for any sequence of processes $\left\{\mathcal{X}^{(x)}(x T), x=1,2, \ldots\right\}$, we have

$$
\limsup _{x \rightarrow \infty} \mathbf{E}\left[\frac{1}{x}\left\|\mathcal{X}^{(x)}(x T)\right\|\right] \leq 1-\epsilon,
$$

then the Markov chain $\mathcal{X}$ is stable.

Lemma 14 implies the stability of the network, and a stability criteria of type (21) leads to a fluid limit approach [17] to the stability problem of queueing systems.

In the following, we construct the fluid limit model of the system as in [13], [17]. We assume that the packets present in the system in its initial state $\mathcal{X}^{(x)}(0)$ arrived in some of the past time-slots $-(x-1),-(x-2), \ldots, 0$, according to their delays in state $\mathcal{X}(0)$. We define another process $\mathcal{Y} \triangleq$ $(A, Q, W, Y)$, i.e., a tuple that denotes a list of process, and clearly, a sample path of $\mathcal{Y}^{(x)}$ uniquely 
defines the sample path of $\mathcal{X}^{(x)}$. Then, we extend the definition of $\mathcal{Y}$ to each continuous time $t \geq 0$ as $\mathcal{Y}^{(x)}(t) \triangleq \mathcal{Y}^{(x)}(\lfloor t\rfloor)$, where $\lfloor t\rfloor$ denotes the integer part of $t$.

Next, we consider a sequence of processes $\left\{\frac{1}{x_{m}} \mathcal{Y}^{\left(x_{m}\right)}\left(x_{m} \cdot\right)\right\}$ that are scaled in both time and space. Then, using the techniques of Theorem 4.1 of [17] or Lemma 1 of [13], we can show that for almost all sample paths and for any sequence of processes $\left\{\frac{1}{x_{m}} \mathcal{Y}^{\left(x_{m}\right)}\left(x_{m} \cdot\right)\right\}$, where $\left\{x_{m}\right\}$ is a sequence of positive integers with $x_{m} \rightarrow \infty$, there exists a subsequence $\left\{x_{m_{l}}\right\}$ with $x_{m_{l}} \rightarrow \infty$ as $l \rightarrow \infty$ such that the following convergences hold uniformly over compact (u.o.c.) interval:

$$
\begin{aligned}
& \frac{1}{x_{m_{l}}} \int_{0}^{x_{m_{l}} t} A_{i}^{\left(x_{m_{l}}\right)}(\tau) d \tau \rightarrow \lambda_{i} t \\
& \frac{1}{x_{m_{l}}} \int_{0}^{x_{m_{l}} t} Y_{i, j}^{\left(x_{m_{l}}\right)}(\tau) d \tau \rightarrow \int_{0}^{t} y_{i, j}(\tau) d \tau, \\
& \frac{1}{x_{m_{l}}} Q_{i}^{\left(x_{m_{l}}\right)}\left(x_{m_{l}} t\right) \rightarrow q_{i}(t) .
\end{aligned}
$$

Similarly, the following convergences (which are denoted by " $\Rightarrow$ ”) hold at every continuous point of the limiting function $w_{i}(t)$ :

$$
\frac{1}{x_{m_{l}}} W_{i}^{\left(x_{m_{l}}\right)}\left(x_{m_{l}} t\right) \Rightarrow w_{i}(t)
$$

Any set of limiting functions $(q, y, w)$ is called a fluid limit. It is easy to show that the limiting functions are Lipschitz continuous in $[0, \infty)$, and are thus absolutely continuous. Therefore, these limiting functions are differentiable at almost all (scaled) time $t \in[0, \infty)$, which we call regular time. Moreover, the limiting functions satisfy that

$$
\sum_{1 \leq i \leq n} q_{i}(0)+\sum_{1 \leq i \leq n} w_{i}(0)=1
$$

and that

$$
\frac{d}{d t} q_{i}(t)= \begin{cases}\lambda_{i}-\sum_{j} y_{i, j}(t), & q_{i}(t)>0 \\ \left(\lambda_{i}-\sum_{j} y_{i, j}(t)\right)^{+}, & q_{i}(t)=0\end{cases}
$$

where $(x)^{+} \triangleq \max (x, 0)$.

We then prove the stability of the fluid limit model using a standard Lyapunov technique. We consider a quadratic Lyapunov function in the fluid limit model of the system, and show that the Lyapunov function has a negative drift when its value is greater than 0 , which implies that the fluid limit model is stable.

Using a similar argument as in [12], [13], we can show that under policy $\mathbf{P}$, there exists a finite time $T_{1}>0$ such that for all $t \geq T_{1}$, we have

$$
q_{i}(t)=\lambda_{i} w_{i}(t)
$$


for all $i$. This linear relation is similar to the Little's law and plays a key role in proving stability of the delay-based schemes. We omit the proof of this linear relation for brevity and refer readers to [12], [13].

Let $V(q(t))$ denote the Lyapunov function defined as

$$
V(q(t)) \triangleq \frac{1}{2} \sum_{i=1}^{n} \frac{q_{i}^{2}(t)}{\lambda_{i}} .
$$

Suppose that $\lambda$ is strictly inside $\Lambda^{*}$, then there exists a vector $\mu \in \mathcal{R}$ such that $\lambda_{i}<\sum_{j=1}^{n} \mu_{i, j}$ for all $i$. Let $\beta$ denote the smallest difference between $\lambda_{i}$ and $\sum_{j=1}^{n} \mu_{i, j}$, i.e., $\beta \triangleq \min _{1 \leq i \leq n}\left(\sum_{j=1}^{n} \mu_{i, j}-\lambda_{i}\right)$. Clearly, we have $\beta>0$. It suffices to show that for any $\zeta_{1}>0$, there exist a $\zeta_{2}>0$ and a finite time $T_{2}>0$ such that for all regular time $t \geq T_{2}, V(q(t)) \geq \zeta_{1}$ implies $\frac{D^{+}}{d t^{+}} V(q(t)) \leq-\zeta_{2}$, where $\frac{D^{+}}{d t^{+}} V(q(t))=\lim _{\delta \downarrow 0} \frac{V(q(t+\delta))-V(q(t))}{\delta}$. Choose any $T_{2} \geq T_{1}$. Since $q(t)$ is differentiable for all regular time $t \geq T_{2}$ such that $V(q(t))>0$, we can obtain the derivative of $V(q(t))$ as

$$
\begin{aligned}
& \frac{D^{+}}{d t^{+}} V(q(t)) \\
& \stackrel{(a)}{=} \sum_{i=1}^{n} \frac{q_{i}(t)}{\lambda_{i}} \cdot\left(\lambda_{i}-\sum_{j=1}^{n} y_{i, j}(t)\right) \\
& \stackrel{(b)}{=} \sum_{i=1}^{n} w_{i}(t) \cdot\left(\lambda_{i}-\sum_{j=1}^{n} \mu_{i, j}(t)\right) \\
& \quad+\sum_{i=1}^{n} w_{i}(t) \cdot\left(\sum_{j=1}^{n} \mu_{i, j}(t)-\sum_{j=1}^{n} y_{i, j}(t)\right), \\
& =\sum_{i=1}^{n} w_{i}(t) \cdot\left(\lambda_{i}-\sum_{j=1}^{n} \mu_{i, j}(t)\right) \\
& \quad+\sum_{j=1}^{n}\left(\sum_{i=1}^{n} w_{i}(t) \mu_{i, j}(t)-\sum_{i=1}^{n} w_{i}(t) y_{i, j}(t)\right),
\end{aligned}
$$

where (a) is from (27), and (b) is from (28) along with a little algebra.

From (28) and (29), we can choose $\zeta_{3}>0$ such that $V(q(t)) \geq \zeta_{1}$ implies $\max _{1 \leq i \leq n} w_{i}(t) \geq \zeta_{3}$. Then, in the final result of $(30)$, we can conclude that the first term is bounded. That is,

$$
\begin{aligned}
& \sum_{i=1}^{n} w_{i}(t) \cdot\left(\lambda_{i}-\sum_{j=1}^{n} \mu_{i, j}(t)\right) \\
& \quad \leq-\zeta_{3} \min _{1 \leq i \leq n}\left(\sum_{j=1}^{n} \mu_{i, j}(t)-\lambda_{i}\right) \\
& \quad \leq-\zeta_{3} \beta \\
& \quad \triangleq-\zeta_{2}<0 .
\end{aligned}
$$


Therefore, we have that $\frac{D^{+}}{d t^{+}} V(q(t)) \leq-\zeta_{2}$ if the second term in the final result of (30) is non-positive. We show this in the following.

Considering the neighborhood around a fixed (scaled) time $t \geq T_{2}$, we define $N \triangleq\left\{\left\lceil x_{m_{l}} t\right\rceil,\left\lceil x_{m_{l}} t\right\rceil+\right.$ $\left.1, \ldots,\left\lfloor x_{m_{l}}(t+\delta)\right\rfloor\right\}$, where $\delta$ is a small positive number and $\left\{x_{m_{l}}\right\}$ is a positive subsequence for which the convergence to the fluid limit holds. We will omit the superscript $\left(x_{m_{l}}\right)$ of the random variables (depending on the choice of the sequence $\left\{x_{m_{l}}\right\}$ ) throughout the rest of the proof for notational convenience (e.g., we use $Q_{i}(t)$ to denote $Q_{i}^{\left(x_{m_{l}}\right)}(t)$ ). We want to show that under policy $\mathbf{P}$, in each time-slot $\tau \in N$, each server $S_{j}$ serves a connected queue $Q_{i(j, \tau)}$ having the largest weight in the fluid limits, i.e., $w_{i(j, \tau)}(t)=$ $L_{j}(\tau) \triangleq \max _{i \in \mathcal{S}_{j}(\tau)} w_{i}(t)$ (recall that $\mathcal{S}_{j}(\tau)=\left\{1 \leq i \leq n \mid C_{i, j}(\tau)=1\right\}$ ). Note that the trivial statement holds if $\mathcal{S}_{j}(\tau)=\emptyset$ or $L_{j}(\tau)=0$. Hence, suppose that $\mathcal{S}_{j}(\tau) \neq \emptyset$ and $L_{j}(\tau)>0$. Consider $r, s \in \mathcal{S}_{j}(\tau)$ such that $w_{s}(t)=L_{j}(\tau)$ and $W_{r}(\tau)=\max _{i \in \mathcal{S}_{j}(\tau)} W_{i}(\tau)$. In other words, $Q_{s}$ is a queue having the largest weight in the fluid limit among all the queues being connected to server $S_{j}$ in time-slot $\tau$, and $Q_{r}$ is a queue having the largest weight in the original discrete-time system among all the queues being connected to server $S_{j}$ in time-slot $\tau$. Note that it is possible that $r=s$. Then, for any time-slot $\tau \in N$, we have that

$$
\begin{aligned}
& W_{i(j, \tau)}\left(\left\lceil x_{m_{l}} t\right\rceil\right) \\
& \stackrel{(a)}{\geq} W_{i(j, \tau)}(\tau)-\left(\left\lfloor x_{m_{l}}(t+\delta)\right\rfloor-\left\lceil x_{m_{l}} t\right\rceil\right) \\
& \stackrel{(b)}{\geq} Z_{r, M}(\tau)-\left(\left\lfloor x_{m_{l}}(t+\delta)\right\rfloor-\left\lceil x_{m_{l}} t\right\rceil\right) \\
& \geq Z_{r, M}(\tau)-W_{r}(\tau)+W_{r}(\tau)-\left(\left\lfloor x_{m_{l}}(t+\delta)\right\rfloor-\left\lceil x_{m_{l}} t\right\rceil\right) \\
& \stackrel{(c)}{\geq} Z_{r, M}(\tau)-W_{r}(\tau)+W_{s}(\tau)-\left(\left\lfloor x_{m_{l}}(t+\delta)\right\rfloor-\left\lceil x_{m_{l}} t\right\rceil\right) \\
& \stackrel{(d)}{\geq} Z_{r, M}(\tau)-W_{r}(\tau)+W_{s}\left(\left\lfloor x_{m_{l}}(t+\delta)\right\rfloor\right) \\
& \quad-2\left(\left\lfloor x_{m_{l}}(t+\delta)\right\rfloor-\left\lceil x_{m_{l}} t\right\rceil\right),
\end{aligned}
$$

where (a) and (d) are due to the fact that the HOL delay cannot increase by more than $\left\lfloor x_{m_{l}}(t+\delta)\right\rfloor-\left\lceil x_{m_{l}} t\right\rceil$ within $\left\lfloor x_{m_{l}}(t+\delta)\right\rfloor-\left\lceil x_{m_{l}} t\right\rceil$ time-slots, (b) is from the property of policy $\mathbf{P}$ satisfying the sufficient conditions, and (c) is due to $W_{r}(\tau)=\max _{i \in \mathcal{S}_{j}(\tau)} W_{i}(\tau)$ and $s \in \mathcal{S}_{j}(\tau)$. Divide both sides of the final 
result of the above equation by $x_{m_{l}}$ and let $x_{m_{l}}$ goes to infinity, we have that

$$
\begin{aligned}
w_{i(j, \tau)}(t) & \stackrel{(a)}{=} \lim _{x_{m_{l}} \rightarrow \infty} \frac{W_{i(j, \tau)}\left(x_{m_{l}} t\right)}{x_{m_{l}}} \\
& \stackrel{(b)}{\geq} \lim _{x_{m_{l}} \rightarrow \infty} \frac{Z_{r, M}(\tau)-W_{r}(\tau)}{x_{m_{l}}}+w_{s}(t+\delta)-2 \delta \\
& \stackrel{(c)}{=} w_{s}(t+\delta)-2 \delta
\end{aligned}
$$

where (a) is from the definition of fluid limits, (b) is from $\underline{31 !}$ and $\lim _{x_{m_{l}} \rightarrow \infty} \frac{\left\lfloor x_{m_{l}}(t+\delta)\right\rfloor-\left\lceil x_{m_{l}} t\right\rceil}{x_{m_{l}}}=\delta$, and (c) is because $\lim _{x_{m_{l}} \rightarrow \infty} \frac{Z_{r, M}(\tau)-W_{r}(\tau)}{x_{m_{l}}}=0$, as the SLLN of (1) holds and $Z_{r, M}(\tau)-W_{r}(\tau)$ is the difference of the arriving times of two packets having finite number of packets in-between. Since the above equation holds for any arbitrarily small positive number $\delta$, by letting $\delta$ go to 0 on both sides of the final result of the above equation, we have $w_{i(j, \tau)}(t) \geq w_{s}(t)=L_{j}(\tau)$, and in particular, we have $w_{i(j, \tau)}(t)=L_{j}(\tau)$. This is true for each $j$ and for each $\tau \in N$. Therefore, under policy $\mathbf{P}$, the service vector $y(t)$ satisfies that

$$
\sum_{i=1}^{n} w_{i}(t) y_{i, j}(t)=\max _{\nu \in \mathcal{R}} \sum_{i=1}^{n} w_{i}(t) \nu_{i, j}
$$

for all $j \in\{1,2, \ldots, n\}$.

Thus, we have that

$$
y(t) \in \underset{\nu \in \mathcal{R}}{\operatorname{argmax}} \sum_{j=1}^{n} \sum_{i=1}^{n} w_{i}(t) \nu_{i, j},
$$

which implies that

$$
\sum_{j=1}^{n} \sum_{i=1}^{n} w_{i}(t) \mu_{i, j}(t) \leq \sum_{j=1}^{n} \sum_{i=1}^{n} w_{i}(t) y_{i, j}(t) .
$$

Therefore, this shows that $V(q(t)) \geq \zeta_{1}$ implies $\frac{D^{+}}{d t^{+}} V(q(t)) \leq-\zeta_{2}$ for all $t \geq T_{2}$. It immediately follows that for any $\zeta>0$, there exists a finite $T \geq T_{2}>0$ such that $\sum_{1 \leq i \leq n} q_{i}(T) \leq \zeta$. Further, we have that

$$
\sum_{1 \leq i \leq n}\left(q_{i}(T)+w_{i}(T)\right) \leq\left(1+\frac{1}{\min _{1 \leq i \leq n} \lambda_{i}}\right) \zeta
$$

due to the linear relation (28).

Now, consider any fixed sequence of processes $\left\{\mathcal{X}^{(x)}, x=1,2, \ldots\right\}$ (for simplicity also denoted by $\{x\}$ ). From the convergences (22)-(25), we have that for any subsequence $\left\{x_{m}\right\}$ of $\{x\}$, there exists a further (sub)subsequence $\left\{x_{m_{l}}\right\}$ such that

$$
\begin{aligned}
\lim _{j \rightarrow \infty} & \frac{1}{x_{m_{l}}}\left\|\mathcal{X}^{\left(x_{m_{l}}\right)}\left(x_{m_{l}} T\right)\right\| \\
\quad & =\sum_{1 \leq i \leq n}\left(q_{i}(T)+w_{i}(T)\right) \leq\left(1+\frac{1}{\min _{1 \leq i \leq n} \lambda_{i}}\right) \zeta
\end{aligned}
$$


almost surely. This in turn implies (for small enough $\zeta$ ) that

$$
\lim _{x \rightarrow \infty} \frac{1}{x}\left\|\mathcal{X}^{(x)}(x T)\right\| \leq\left(1+\frac{1}{\min _{1 \leq i \leq n} \lambda_{i}}\right) \zeta \triangleq 1-\epsilon<1
$$

almost surely.

We can show that the sequence $\left\{\frac{1}{x}\left\|\mathcal{X}^{(x)}(x T)\right\|, x=1,2, \ldots\right\}$ is uniformly integrable, due to the following:

$$
\frac{1}{x}\left\|\mathcal{X}^{(x)}(x T)\right\| \leq 1+\frac{1}{x} \sum_{1 \leq i \leq n} \int_{\tau=0}^{x T} A_{i}(\tau) d \tau+n T
$$

and

$$
\mathbf{E}\left[1+\frac{1}{x} \sum_{1 \leq i \leq n} \int_{\tau=0}^{x T} A_{i}(\tau) d \tau+n T\right]<\infty,
$$

where the above finite expectation is from our assumption on the arrival process. Then, the almost surely convergence in (35) along with uniform integrability implies the following convergence in the mean:

$$
\limsup _{x \rightarrow \infty} \mathbf{E}\left[\frac{1}{x}\left\|\mathcal{X}^{(x)}(x T)\right\|\right] \leq 1-\epsilon .
$$

Since the above convergence holds for any sequence of processes $\left\{\mathcal{X}^{(x)}(x T), x=1,2, \ldots\right\}$, the condition of type (21) in Lemma 14 is satisfied. This completes the proof of Theorem 6

\section{APPENDIX H}

\section{Proof of Proposition 7}

We prove it by showing that DWM is an MWF policy.

Let $M=n$. We want to show that the sufficient condition in Theorem 6 is satisfied, i.e., in any time-slot $t$ and for all $j \in\{1,2, \ldots, n\}$, DWM policy allocates server $S_{j}$ to serve queue $Q_{i(j, t)}$, which satisfies that $W_{i(j, t)}(t) \geq Z_{r, n}(t)$ for all $r \in \Gamma_{j}(t)$ such that $Q_{r}(t) \geq n$.

Suppose that the sufficient condition is not satisfied, i.e., consider any server $S_{j}$ such that $Q_{r}(t) \geq n$ for some $r \in \Gamma_{j}(t)$, and $S_{j}$ is allocated to serve queue $Q_{i(j, t)}$, and suppose that $W_{i(j, t)}(t)<Z_{r, n}(t)$. Since $Q_{r}(t) \geq n$ and at most $n-1$ packets could be matched with the other $n-1$ servers, there must be at least one of the $n$ oldest packets in $Q_{r}$ remaining unmatched. Suppose this packet is the $k$-th oldest packet in queue $Q_{r}$, then $Z_{r, k}(t) \geq Z_{r, n}(t)>W_{i(j, t)}(t)$. Hence, DWM policy must match $S_{j}$ to the $k$-th oldest packet in queue $Q_{r}$, i.e., DWM must allocate $S_{j}$ to serve $Q_{r}$ rather than $Q_{i(j, t)}$, which is a contradiction.

Therefore, DWM policy is an MWF policy and is thus throughput-optimal. 


\section{APPENDIX I}

\section{Proof of Proposition 8}

By an argument similar to that in Theorem 3 of [7], we want to show that under D-MWS, the delayviolation event occurs with at least a constant probability for any fixed delay threshold even if $n$ is large.

First, we define $D(x \| y) \triangleq x \log \frac{x}{y}+(1-x) \log \frac{1-x}{1-y}$. Then, fix any real number $p^{\prime} \in(0, p)$ and any integer $T$, and consider any configuration of queues at the end of time-slot $T$.

In time-slot $T+1$ :

By the Chernoff bound, there exists an integer $N_{1}$ such that for all $n \geq N_{1}$, with probability at least $1-e^{-D\left(p^{\prime}|| p\right) n}$, at least $n p^{\prime}$ queues have packet arrivals at the beginning of time-slot $T+1$. Define $\mu \triangleq-2 / \log (1-q)$ and $\nu \triangleq \mu \log n$. Fix an integer $N_{2}$ such that for all $n \geq N_{2}$, we have $\nu \geq 1$. Sort the queues in the order of priority for service under D-MWS, i.e., after sorting, the first queue has the largest weight (HOL delay) with the smallest index; the second queue has the largest weight with the second smallest index, or has the second largest weight with the smallest index if there is only one queue having the largest weight; and so on. Let the set of the first $\nu$ queues after sorting be $\mathcal{Q}^{*} \triangleq\left\{Q_{i_{1}}, Q_{i_{2}}, \ldots, Q_{i_{\nu}}\right\}$. Let $E_{j}$ denote the event that server $S_{j}$ is not connected with any of the queues in $\mathcal{Q}^{*}$. Then, $\mathbb{P}\left(E_{j}\right)=(1-q)^{\nu}=(1-q)^{\mu \log n}$, and we have that

$$
\mathbb{P}\left(\cup_{j=1}^{n} E_{j}\right) \leq \sum_{j=1}^{n} \mathbb{P}\left(E_{j}\right)=n(1-q)^{\mu \log n}=\frac{1}{n},
$$

where the last equality is because $(1-q)^{\mu \log n}=\exp (\mu \log n \log (1-q))=\frac{1}{n^{2}}$. Thus, with probability at least $1-\frac{1}{n}$, each server is connected to at least one queue in $\mathcal{Q}^{*}$. According to the operations of D-MWS, a server connected to at least one queue in $\mathcal{Q}^{*}$ must be allocated to one of the queues in $\mathcal{Q}^{*}$. Hence, with probability at least $1-\frac{1}{n}$, all the servers serve queues in $\mathcal{Q}^{*}$. Since $\left|\mathcal{Q}^{*}\right|=\nu$ and with probability at least $1-e^{-D\left(p^{\prime} \| p\right) n}$, at least $n p^{\prime}$ queues had packet arrivals, it follows that for $n \geq N_{3} \triangleq \max \left\{N_{1}, N_{2}\right\}$, with probability at least $1-\frac{1}{n}-e^{-D\left(p^{\prime}|| p\right) n}$ by the union bound, at the end of time-slot $T+1$ (and at the beginning of time-slot $T+2$ ), the system has at least $n p^{\prime}-\nu$ queues having a weight (HOL delay) of at least 1 . Let this set of queues (of weight being 1) be $\mathcal{A}_{1}$.

In time-slot $T+2$ :

By the similar argument above for time-slot $T+1$, it follows that, with probability at least $1-\frac{1}{n}$, no more than $\nu$ queues can receive service. Combining this with the result for time-slot $T+1$ and using the union bound, we have that for all $n \geq N_{3}$, with probability at least $1-\frac{2}{n}-e^{-D\left(p^{\prime}|| p\right) n}$, at the end 
of time-slot $T+2$ (and at the beginning of time-slot $T+3$ ), there exists a set $\mathcal{A}_{2}$ of queues such that $\left|\mathcal{A}_{2}\right| \geq\left|\mathcal{A}_{1}\right|-\nu \geq n p^{\prime}-2 \nu$, and each queue in $\mathcal{A}_{2}$ has a weight (HOL delay) of at least 2.

Repeating the same argument above, we have that for all $n \geq N_{3}$, with probability at least $1-\frac{b+1}{n}-$ $e^{-D\left(p^{\prime}|| p\right) n}$, at the end of time-slot $T+b+1$, there exists a set $\mathcal{A}_{b+1}$ of queues such that $\left|\mathcal{A}_{b+1}\right| \geq$ $n p^{\prime}-(b+1) \nu$, and each queue in $\mathcal{A}_{b+1}$ has a weight (HOL delay) of at least $b+1$.

Fix a real number $\epsilon \in(0,1)$, there exists an integer $N_{4}$ such that for all $n \geq N_{4}$, we have $1-$ $\frac{b+1}{n}-e^{-D\left(p^{\prime}|| p\right) n} \geq \epsilon$ and $n p^{\prime}-(b+1) \nu=n p^{\prime}-(b+1) \mu \log n \geq 1$. Hence, for a system with

$n \geq N_{5} \triangleq \max \left\{N_{3}, N_{4}\right\}$, starting with time-slot $T$, with probability at least $\epsilon$, we have at least one queue having a HOL delay of at least $b+1$ at the end of time-slot $T+b+1$ (or at the beginning of time-slot $T+b+2$ ). Let $T=-b-2$, then the above result shows that the delay violation event occurs with at least a constant probability even if $n$ is large. This completes the proof.

\section{APPENDIX J}

\section{ProOF OF THEOREM 9}

We first show that a hybrid OPF-MWF policy is an (overall) OPF policy and is thus rate-function delay-optimal. Note that in stage 1, the operations of an OPF policy already guarantees that the sufficient condition in Theorem 2 is satisfied. Since in stage 2, the matched servers and packets in stage 1 will not be considered, it ensures that the operations do not perturb the satisfaction of the sufficient condition for rate-function delay optimality.

In the following, we want to show that a hybrid OPF-MWF policy is an (overall) MWF policy and is thus throughput-optimal. Let $M=n$. We want to show that the sufficient condition in Theorem 6 is satisfied, i.e., in any time-slot $t$ and for all $j \in\{1,2, \ldots, n\}$, a hybrid OPF-MWF policy allocates server $S_{j}$ to serve queue $Q_{i(j, t)}$, which satisfies that $W_{i(j, t)}(t) \geq Z_{r, n}(t)$ for all $r \in \Gamma_{j}(t)$ such that $Q_{r}(t) \geq n$.

First, we want to show that in stage 1 , an OPF policy also guarantees that all allocated servers in stage 1 satisfies the sufficient condition for throughput optimality. Consider each server $S_{l}$ such that $l \in\{1,2, \ldots, n\} \backslash R(t)$, i.e., all servers $S_{j}$ that are allocated in stage 1 . Then, $Q_{i(l, t)}$ is the queue served by server $S_{l}$ in stage 1 of time-slot $t$. Since we run an OPF policy in stage 1 , server $S_{l}$ serves a packet among the $n$ oldest packets in the system, and it must satisfy that $W_{i(j, t)}(t) \geq Z_{r, n}(t)$ for any $r \in \Gamma_{l}(t)$ such that $Q_{r}(t) \geq n$.

Next, consider each server $S_{j}$ such that $j \in R(t)$, then $Q_{i(j, t)}$ is the queue served by server $S_{j}$ in stage 2 of time-slot $t$. It is clear from Condition 2) of Definition 3 that $W_{i(j, t)}(t) \geq Z_{r, n}(t)$ for all $r \in \Gamma_{j}(t)$ such that $Q_{r}(t) \geq n$. 
Therefore, a hybrid OPF-MWF policy is an (overall) MWF policy and is thus throughput-optimal.

\section{APPENDIX K}

\section{PROOF OF THEOREM 10}

To show that DWM- $n$-MWS is a hybrid OPF-MWF policy, it is sufficient to show that Condition 2) of Definition 3 is satisfied.

Given any time-slot $t$, consider each server $S_{j}$ such that $j \in R(t)$, then $Q_{i(j, t)}$ is the queue served by server $S_{j}$ in stage 2 under D-MWS. Let $M=n$. We want to show that $W_{i(j, t)}(t) \geq Z_{r, n}(t)$ for all $r \in \Gamma_{j}(t)$ such that $Q_{r}(t) \geq n$.

Let $W_{i}^{\prime}(t)$ be the HOL delay of queue $Q_{i}$ at the beginning of stage 2 . Let $\Gamma_{j}^{\prime}(t)$ denote the set of queues that are connected to server $S_{j}$ and have the largest weight among the connected queues at the beginning of stage 2 of time-slot $t$, i.e., $\Gamma_{j}^{\prime}(t) \triangleq\left\{i \in \mathcal{S}_{j}(t) \mid W_{i}^{\prime}(t)=\max _{l \in \mathcal{S}_{j}(t)} W_{l}^{\prime}(t)\right\}$, where $\mathcal{S}_{j}(t)=\left\{1 \leq i \leq n \mid C_{i, j}(t)=1\right\}$. According to the operations of D-MWS, the index of queue that is served by server $S_{j}$ satisfies that $i(j, t)=\min \left\{i \mid i \in \Gamma_{j}^{\prime}(t)\right\}$, hence, we have $W_{i(j, t)}^{\prime}(t)=W_{r}^{\prime}(t)$ for any $r \in \Gamma_{j}^{\prime}(t)$. This implies that $W_{i(j, t)}(t) \geq W_{i(j, t)}^{\prime}(t)=W_{r}^{\prime}(t) \geq Z_{r, n}(t)$ for any $r \in \Gamma_{j}^{\prime}(t)$ such that $Q_{r}(t) \geq n$, where the last inequality is because $Q_{r}(t) \geq n$ and thus the HOL packet of queue $Q_{r}$ at the beginning of stage 2 must not have a later position than the $n$-th packet in queue $Q_{r}$ at the beginning of time-slot $t$. This holds for all $j \in R(t)$ and any time-slot $t$. Therefore, DWM- $n$-MWS is a hybrid OPF-MWF policy.

Since the complexity of DWM- $n$ and D-MWS is $O\left(n^{2.5} \log n\right)$ and $O\left(n^{2}\right)$, respectively, the overall complexity of DWM- $n$-MWS policy is $O\left(n^{2.5} \log n\right)$.

\section{REFERENCES}

[1] D. Shah, D. N. C. Tse, and J. N. Tsitsiklis, "Hardness of low delay network scheduling," IEEE Transactions on Information Theory, vol. 57, no. 12, pp. 7810-7817, 2011.

[2] L. Tassiulas and A. Ephremides, "Dynamic server allocation to parallel queues with randomly varying connectivity," IEEE Transactions on Information Theory, vol. 39, no. 2, pp. 466-478, 1993.

[3] A. Ganti, E. Modiano, and J. N. Tsitsiklis, "Optimal transmission scheduling in symmetric communication models with intermittent connectivity," IEEE Transactions on Information Theory, vol. 53, no. 3, pp. 998-1008, 2007.

[4] S. Kittipiyakul and T. Javidi, "Delay-optimal server allocation in multiqueue multiserver systems with time-varying connectivities," IEEE Transactions on Information Theory, vol. 55, no. 5, pp. 2319-2333, 2009.

[5] — , "Resource allocation in OFDMA with time-varying channel and bursty arrivals," IEEE Communications Letters, vol. 11, no. 9, pp. 708-710, 2007. 
[6] S. Bodas, S. Shakkottai, L. Ying, and R. Srikant, "Scheduling in multi-channel wireless networks: Rate function optimality in the small-buffer regime," in ACM Proceedings of the eleventh international joint conference on Measurement and modeling of computer systems (SIGMETRICS), 2009, pp. 121-132.

[7] — - "Low-complexity scheduling algorithms for multi-channel downlink wireless networks," in The IEEE International Conference on Computer Communications (INFOCOM). IEEE, 2010, pp. 1-9.

[8] _ _ "Scheduling for small delay in multi-rate multi-channel wireless networks," in The IEEE International Conference on Computer Communications (INFOCOM). IEEE, 2011, pp. 1251-1259.

[9] S. Bodas and T. Javidi, "Scheduling for multi-channel wireless networks: Small delay with polynomial complexity," in 2011 International Symposium on Modeling and Optimization in Mobile, Ad Hoc and Wireless Networks (WiOpt). IEEE, 2011, pp. 78-85.

[10] M. Sharma and X. Lin, "OFDM downlink scheduling for delay-optimality: Many-channel many-source asymptotics with general arrival processes," Purdue University, Tech. Rep., 2011. [Online]. Available: https://engineering.purdue.edu/\% 7elinx/papers.html

[11] _ _ "OFDM downlink scheduling for delay-optimality: Many-channel many-source asymptotics with general arrival processes," in The IEEE Information Theory and Applications Workshop (ITA), 2011.

[12] B. Ji, C. Joo, and N. B. Shroff, "Delay-Based Back-Pressure Scheduling in Multihop Wireless Networks," IEEE/ACM Transactions on Networking, vol. 21, no. 5, pp. 1539-1552, 2013.

[13] M. Andrews, K. Kumaran, K. Ramanan, A. Stolyar, R. Vijayakumar, and P. Whiting, "Scheduling in a queuing system with asynchronously varying service rates," Probability in the Engineering and Informational Sciences, vol. 18, pp. 191-217, 2004.

[14] M. Fredman and R. Tarjan, "Fibonacci heaps and their uses in improved network optimization algorithms," Journal of the ACM (JACM), vol. 34, no. 3, pp. 596-615, 1987.

[15] T. Spencer and E. Mayr, "Node weighted matching," Automata, Languages and Programming, pp. 454-464, 1984.

[16] G. Gupta, S. Sanghavi, and N. Shroff, "Node weighted scheduling," in ACM Proceedings of the eleventh international joint conference on Measurement and modeling of computer systems (SIGMETRICS), 2009, pp. 97-108.

[17] J. Dai, "On positive Harris recurrence of multiclass queueing networks: a unified approach via fluid limit models," The Annals of Applied Probability, pp. 49-77, 1995.

[18] L. Tassiulas and A. Ephremides, "Stability properties of constrained queueing systems and scheduling policies for maximum throughput in multihop radio networks," IEEE Transactions on Automatic Control, vol. 37, no. 12, pp. 1936-1948, 1992.

[19] X. Lin, N. B. Shroff, and R. Srikant, "A tutorial on cross-layer optimization in wireless networks," IEEE Journal on Selected Areas in Communications, vol. 24, no. 8, pp. 1452-1463, Aug. 2006.

[20] L. Georgiadis, M. Neely, M. Neely, and L. Tassiulas, "Resource allocation and cross-layer control in wireless networks," Foundations and Trends in Networking, vol. 1, no. 1, pp. 1-144, 2006.

[21] A. Eryilmaz, R. Srikant, and J. Perkins, "Stable scheduling policies for fading wireless channels," IEEE/ACM Transactions on Networking, vol. 13, no. 2, pp. 411-424, 2005.

[22] S. P. Boyd and L. Vandenberghe, Convex optimization. Cambridge university press, 2004.

[23] T. Cormen, C. Leiserson, R. Rivest, and C. Stein, Introduction to Algorithms, 3rd ed. The Massachusetts Institute of Technology, 2009.

[24] A. Rybko and A. Stolyar, "Ergodicity of stochastic processes describing the operation of open queueing networks," Problems of Information Transmission, vol. 28, pp. 199-220, 1992. 
[25] V. Malyshev and M. Menshikov, "Ergodicity, continuity and analyticity of countable Markov chains," Transactions of the Moscow Mathematical Society, vol. 39, pp. 3-48, 1979. 